\title{
Buckling Transitions and Clock Order of Two-Dimensional Coulomb Crystals
}

\author{
Daniel Podolsky, ${ }^{1}$ Efrat Shimshoni, ${ }^{2}$ Giovanna Morigi, ${ }^{3}$ and Shmuel Fishman ${ }^{1}$ \\ ${ }^{1}$ Department of Physics, Technion, Haifa 32000, Israel \\ ${ }^{2}$ Department of Physics, Bar-Ilan University, Ramat-Gan 52900, Israel \\ ${ }^{3}$ Theoretische Physik, Universität des Saarlandes, D-66123 Saarbrücken, Germany \\ (Received 2 December 2015; revised manuscript received 16 June 2016; published 22 August 2016)

\begin{abstract}
Crystals of repulsively interacting ions in planar traps form hexagonal lattices, which undergo a buckling instability towards a multilayer structure as the transverse trap frequency is reduced. Numerical and experimental results indicate that the new structure is composed of three planes, whose separation increases continuously from zero. We study the effects of thermal and quantum fluctuations by mapping this structural instability to the six-state clock model. A prominent implication of this mapping is that at finite temperature, fluctuations split the buckling instability into two thermal transitions, accompanied by the appearance of an intermediate critical phase. This phase is characterized by quasi-long-range order in the spatial tripartite pattern. It is manifested by broadened Bragg peaks at new wave vectors, whose line shape provides a direct measurement of the temperature-dependent exponent $\eta(T)$ characteristic of the power-law correlations in the critical phase. A quantum phase transition is found at the largest value of the critical transverse frequency: Here, the critical intermediate phase shrinks to zero. Moreover, within the ordered phase, we predict a crossover from classical to quantum behavior, signifying the emergence of an additional characteristic scale for clock order. We discuss experimental realizations with trapped ions and polarized dipolar gases, and propose that within accessible technology, such experiments can provide a direct probe of the rich phase diagram of the quantum clock model, not easily observable in condensed matter analogues. Therefore, this work highlights the potential for ionic and dipolar systems to serve as simulators for complex models in statistical mechanics and condensed matter physics.
\end{abstract}

DOI: 10.1103/PhysRevX.6.031025

\section{INTRODUCTION}

The statistical physics of two-dimensional (2D) systems is fascinating, as it exhibits phases that are critical for a nonvanishing range of parameters. In these phases, there is no true long-range order, and correlations decay as a power law. The pioneering work of Kosterlitz and Thouless [1] and of Berezinskii [2] laid the foundation for the theoretical analysis of such systems. This critical behavior requires the symmetry of the Hamiltonian with respect to rotation of the order parameter by some angle variable $\theta$. Its physics is therefore captured by an easy-plane spin Hamiltonian known as the $X Y$ model.

An interesting situation may be encountered if the planar rotation symmetry is broken by an additional term in the Hamiltonian, which favors specific evenly spaced values of the angle $\theta_{i}=2 \pi i / q$, for $i=1,2, \ldots, q$. The resulting model is dubbed the $q$-state $\left(Z_{q}\right)$ clock model. At low enough temperatures, the phases get pinned at one of the favored discrete values $\theta_{i}$, signaling the onset of true

Published by the American Physical Society under the terms of the Creative Commons Attribution 3.0 License. Further distribution of this work must maintain attribution to the author(s) and the published article's title, journal citation, and DOI.
Subject Areas: Condensed Matter Physics, Magnetism, Statistical Physics long-range order. Interestingly, for $q>4$, the thermal transition between ordered and disordered phases is separated by an intermediate phase with critical correlations [3]. At zero temperature, quantum fluctuations take over. Then, the ordered and disordered phases are directly connected by a quantum critical point [4-7].

The unique features of the $X Y$ and clock models in 2D are manifested in a broad and expanding set of distinct physical systems. The most familiar realizations of the 2D $X Y$ model are associated with superfluidity and superconductivity in thin films, where several predictions were verified experimentally [8-10], and in closely analogous systems like melting of two-dimensional crystals [11-14] and dusty plasmas [15]. More recently, features of the Kosterlitz-Thouless transitions have been revealed in coldatom experiments with Bose-Einstein condensates in two dimensions [16,17], including the observation of vortex proliferation in two-dimensional Josephson arrays [18]. Realizations of the clock model were also discussed in a variety of contexts; for example, supersolid order of hardcore bosons in triangular lattices was predicted in Refs. [19-21]. This type of model provides a good description for some frustrated quantum magnet systems, where a two-step melting of the six-state clock order via an intermediate, critical phase was recently discussed [22]. We 
further note that the physics of the clock model and the existence of an intermediate phase have an analogue in lattice gauge theories in higher dimensions [23,24], where the critical phase corresponds to quantum electrodynamics with massless photons.

In the present paper, this rich critical behavior is studied in the framework of a concrete model of experimental relevance, namely, planar crystals of strongly interacting atoms, with a particular focus on realizations with trapped ions. Ion Coulomb crystals are an example of ordered states of matter which emerge from the competition between kinetic energy and repulsive forces in confined volumes $[25,26]$. Their realization in atomic physics laboratories opens the possibility to gain insight into the dynamics of diverse physical models, such as Wigner crystals [27] of electrons on the surface of liquid helium [28], white dwarf cores, and neutron-star outer crusts $[29,30]$. Thanks to the advances in trapping and cooling [31], different structures can be realized by tuning the strength of the external confining potential. The different structural order results from the interplay between the repulsive interactions between the ions and the trap geometry at low thermal energies and has been extensively analyzed in the classical regime by means of numerical simulations $[26,32,33]$ and of impressive experimental realizations [34-38].

The manifestation of statistical mechanics and fieldtheoretical models in ion crystal systems has already been discussed in the context of one-dimensional ion chains. For instance, the mechanical instability of a linear chain of ions to a zigzag structure can be classically mapped to a Landau model [39], and the field-theoretical model predicts a quantum phase transition of the Ising universality class [40-42]. Careful numerical analysis based on DMRG-type programs evaluated the quantum critical exponents with high precision and showed that the quantum critical region has a very small size because of the heavy ion mass [42-44].

The transverse instability of planar lattices of ions, by contrast, is largely unexplored and offers the possibility of observing much richer physics. These two-dimensional structures are presently analyzed as platforms for quantum information processing $[45,46]$. Early theory work by Dubin predicted a buckling instability manifested as a continuous phase transition to a three-plane structure [32]. Similar results were obtained for a crystal of atoms interacting with a repulsive dipolar interaction [47]. Features hinting at the formation of three planes were measured in trapped ions experiments, even though these could not be revealed by Bragg scattering [36,37]. The predicted structure is illustrated in Fig. 1.

Intriguing properties are to be expected when fluctuations are taken into account. The buckling instability in Fig. 1 can be described by a complex-valued order parameter $\left(\psi=|\psi| e^{i \theta}\right)$, namely, the Fourier transform of the height configuration, evaluated at the wave vector $\mathbf{K}$ of

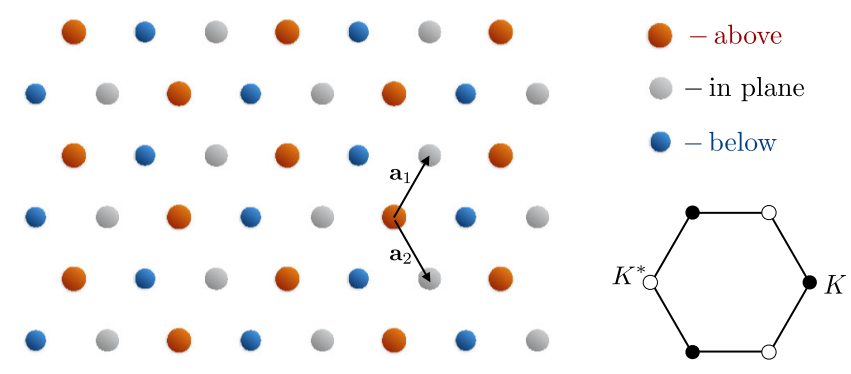

FIG. 1. Ions in a planar trap form a hexagonal lattice, which undergoes a mechanical instability as a function of the temperature and/or the transverse frequency. Left panel: Height pattern in the ordered phase. The hexagonal lattice is split into three sublattices, one of which stays in the $z=0$ plane (gray circles), one is raised above it (red), and one is lowered below it (blue). There are six inequivalent configurations, corresponding to the 3 ! possible height assignments to the sublattices. Right panel: The first Brillouin zone of the undistorted hexagonal lattice is shown, together with the wave vectors $\mathbf{K}$ and $\mathbf{K}^{*}=-\mathbf{K}$ corresponding to the height pattern.

the tripartite order. Fluctuations in the phase $\theta$ of the order parameter are captured by the $X Y$ model. At yet lower temperatures, the phase $\theta$ is expected to be pinned to one of six favored discrete values $\theta_{i}$, leading to true long-range order and spontaneous breaking of $Z_{6}$ symmetry. In this work, we argue that the planar-to-buckled instability is captured by the six-state clock model $[3,23,48]$.

As already noted above, the six-state clock model predicts the existence of two transition lines at finite temperatures. This behavior follows from a duality relation between the fully ordered (low- $T$ ) and fully disordered (high- $T$ ) phases $[23,48]$, dictating two distinct critical temperatures $\left(T_{l}, T_{h}\right)$ at opposite sides of the self-dual point. It implies the formation of an intermediate, critical phase with power-law correlations in the finite range of temperatures, $T_{l}<T<T_{h}$. In our case, this physics is manifested as follows: At fixed transverse trap frequency, there exists a temperature $T_{\mathrm{KT}}$ below which the planar crystal becomes unstable, as shown in the phase diagram in Fig. 2. Here, the ions' transverse displacements fluctuate between three layers and exhibit a buckled pattern with quasi-long-range order. At a second temperature $T_{6}$, below $T_{\mathrm{KT}}$, true long-range order is established, leading to the formation of three phase-locked layers (see Fig. 1). The values of $T_{\mathrm{KT}}$ and $T_{6}$, and hence the size of the critical intermediate phase, depend on a parameter $r$ tuned by the trap frequency and are shown to vanish at a single quantum critical point at $T=0$. By the general theory of quantum phase transitions [49], here the model is in the universality class of the $X Y$ model in $\mathrm{D}=2+1$ dimensions, up to dangerously irrelevant perturbations [4] (for the formal definition, see Ref. [50]). Finally, for $T$ finite but sufficiently small, we predict a crossover within the ordered phase from classical to quantum behavior at a temperature $T_{6}^{*}<T_{6}$. This signifies the emergence of an additional 


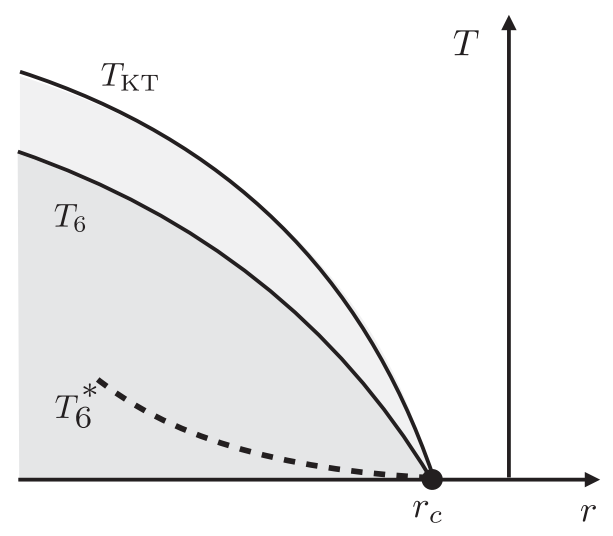

FIG. 2. Phase diagram of the structural instability of a planar ion crystal in the parameter space determined by the temperature $T$ and the coefficient $r$, which is a function of the ratio $\mathcal{K} / C$. The external line at $T_{\mathrm{KT}}$ indicates the transition from a planar structure to a critical phase, where the ions are distributed along three layers and exhibit quasi-long-range order in the transverse direction. The second line at $T_{6}$ indicates the transition to a long-range ordered buckled phase, where the ions form three hexagonal lattices in the three layers, which are phase locked as shown in Fig. 1. The value $r_{c}<0$ indicates the critical point at $T=0$. The dashed line at $T_{6}^{*}$ separates the quantum from the classical critical behaviors. See text for further details.

characteristic scale for clock order, $\xi_{6} \sim 1 / T_{6}^{*}$, which is intimately related to the clock term being dangerously irrelevant [5].

As we show in this work, the realization of the quantum clock model in cold ion systems provides unique access to features that are typically inaccessible in standard condensed-matter systems (e.g., magnetic compounds). In standard condensed-matter systems, one usually measures global properties, such as susceptibilities and conductivities. By contrast, ionic traps and cold atomic systems allow for the measurement of local properties through direct imaging and also offer continuous control of quantum parameters. In particular, a remarkable feature of the realization we propose in trapped ions is that the different phases separated by the buckling transitions are signaled by specific features of the Bragg diffraction patterns, measurements which are typically accessible in these systems [35]. The line shape of the peaks, for example, provides a direct measurement of the $T$-dependent exponent $\eta(T)$ characteristic of the quasi-ordered Kosterlitz-Thouless phase. In addition, we show that by analyzing the shape of the transition lines, the quantum critical properties can be probed significantly far from the quantum critical point. We further argue that these features can be observed in polarized dipolar gases [51,52], where the quantum critical behavior may be manifested more prominently.

The outline of the paper is as follows: In Sec. II, we introduce the order parameter field and derive the fieldtheoretical model; technical details of the derivation are included in Appendix A. In Sec. III, we analyze the phase diagram. In Sec. IV (supplemented by Appendix B), we derive the dependence of observable physical quantities in the ordered phase on $T$ and the parameters of the model, particularly analyzing the signature of the classicalquantum crossover mentioned above. Our main conclusions are summarized in Sec. V.

\section{DERIVATION OF A FIELD-THEORETICAL MODEL}

We consider a system consisting of repulsively interacting ions confined by a potential to move in the $x y$ plane and its vicinity. The ions form a triangular lattice of spacing $a$ on the plane, as shown in Fig. 1. We assume that their only freedom is to move a small distance in the direction perpendicular to the lattice. This relies on the assumption, supported by numerical data $[32,47]$, that the out-of-plane zigzag instability preempts a structural phase transition in the plane, e.g., from a triangular to a square lattice. Throughout this paper, we focus our discussion on ions, but these considerations can be extended to ultracold dipolar atoms or molecules, as we argue in Sec. II E.

Note that throughout the paper (and unless otherwise stated), we work with units for which the Boltzmann and Planck constants are $k_{B}=\hbar=1$. All length scales are in units of the lattice constant $a$.

\section{A. Two-dimensional ion crystals}

Ions of charge $Q$ and mass $M$ are confined in the $x y$ plane by an anisotropic potential, whose strength is sufficiently large to overcome their mutual repulsion. We assume that the ion density $n$ on the plane is homogeneous and denote by $U_{c}$ the potential confining the ions along the $z$ direction. The potential is harmonic and reads

$$
U_{c}=\frac{C}{2} \sum_{i} z_{i}^{2}
$$

where $z_{j}$ is the dimensionless displacement of ion $j$ from the plane (in units of the lattice spacing $a$ ), $C$ is a parameter with the dimensions of an energy: $C=M \omega_{z}^{2} a^{2}$, and $\omega_{z}$ is the harmonic-trap frequency. When the thermal energy is well below the Coulomb interaction energy, the ions crystallize in a triangular lattice [25,26,33]. In finite systems, this is observed at a sufficient distance from the edges [36,37]. We denote by $\mathbf{r}_{\mathbf{j}}=\left(x_{j}, y_{j}\right)$ the ions' positions on the plane, given in units of $a$. These are the solutions that minimize the in-plane potential energy $U_{Q}^{x y}$ given by

$$
U_{Q}^{x y}=\frac{\mathcal{K}}{2} \sum_{i \neq j} \frac{1}{\sqrt{\left(x_{i}-x_{j}\right)^{2}+\left(y_{i}-y_{j}\right)^{2}}},
$$

where $\mathcal{K}$ is the energy scale of the Coulomb interaction, 


$$
\mathcal{K}=\frac{Q^{2}}{4 \pi \epsilon_{0} a},
$$

with $\epsilon_{0}$ the vacuum permittivity.

In order to set up some notation, we consider a $2 \mathrm{D}$ hexagonal lattice with lattice constant $a=1$ and lattice vectors $\mathbf{a}_{1}$ and $\mathbf{a}_{2}$, given by

$$
\mathbf{a}_{1,2}=(1 / 2, \pm \sqrt{3} / 2) .
$$

A general lattice vector can be written as

$$
\mathbf{r}_{i}=n_{1} \mathbf{a}_{1}+n_{2} \mathbf{a}_{2},
$$

with $n_{\{1,2\}}$ integers. The first Brillouin zone is a hexagon, with two inequivalent corners at the points $\pm \mathbf{K}$, where

$$
\mathbf{K}=(4 \pi / 3,0) .
$$

The other corner points are related to $\pm \mathbf{K}$ by reciprocal lattice vectors.

At fixed planar densities, the planar lattice is unstable against fluctuations in the $z$ directions, which tend to minimize the potential energy. This instability occurs at a critical value of the transverse potential, which depends on $\mathcal{K}$ and has been determined by means of a stability analysis based on minimization of the interaction energy in Ref. [32]. For the purpose of our study, we consider the interparticle potential $U_{Q}$ resulting from the Coulomb repulsion after subtracting $U_{Q}^{x y}$ :

$U_{Q}=\frac{\mathcal{K}}{2} \sum_{i \neq j} \frac{1}{\sqrt{\left(x_{i}-x_{j}\right)^{2}+\left(y_{i}-y_{j}\right)^{2}+\left(z_{i}-z_{j}\right)^{2}}}-U_{Q}^{x y}$,

and derive an effective field-theoretical model assuming small displacements $\left|z_{j}\right| \ll 1$ from the plane at $z=0$.

\section{B. Planar instability and order parameter}

As the confining potential is reduced (or the planar density is increased), the system undergoes a structural transition, in which some of the atoms leave the $z=0$ plane in order to reduce their mutual repulsion. In the energetically favored configuration, the hexagonal lattice divides into a tripartite lattice. This is indicated by direct minimization of the energy in numerical calculations $[32,47]$ and by analytical studies based on the Taylor expansion of the potential in Eq. (2.7) to second order in the height $z$, which we report in Appendix A. Particles on one sublattice rise, $z>0$, on another they are submerged, $z<0$, and on the third they remain level at $z=0$, as shown in Fig. 1. Such a configuration is described by the height $z_{i}$ of the particle at the location $\mathbf{r}_{i}$ and can be succinctly written as

$$
z_{i}=\operatorname{Re}\left[\psi e^{i \mathbf{K} \cdot \mathbf{r}_{i}}\right]
$$

where $\mathbf{K}$ is the vector defined in Eq. (2.6), and $\psi=|\psi| e^{i \theta}$ is a complex number that acts as the order parameter. Then, since

$$
\mathbf{K} \cdot \mathbf{r}_{i}=2 \pi\left(n_{1}+n_{2}\right) / 3,
$$

the requirement that one of the three sublattices remains level at $z=0$ implies that the phase of $\psi$ can only take one of six values at the minimal energy configuration,

$$
\psi=|\psi| e^{i \theta}=|\psi| e^{i \pi(2 n+1) / 6},
$$

where $n \in\{1, \ldots, 6\}$. This suggests that the structural transition may be described in terms of a six-state clock model [3] and is corroborated by a symmetry analysis, as follows.

The hexagonal lattice is symmetric under a number of transformations, including translations, reflections, and rotations. These transformations act on both the lattice positions $\mathbf{r}_{i}$ and on the heights $z_{i}$. Equivalently, using Eq. (2.8), these transformations can be thought of as acting on the order parameter $\psi$. For example, a reflection about the $z=0$ plane, $R_{z}$, changes the sign of the heights $z_{i} \rightarrow$ $-z_{i}$ without affecting the positions $\mathbf{r}_{i}$. This is equivalent to flipping the sign of $\psi$,

$$
R_{z}: \psi \rightarrow-\psi
$$

On the other hand, a translation $T_{\mathbf{a}_{1}}$ by lattice vector $\mathbf{a}_{1}$ can be absorbed into a phase shift of $\psi$ since

$$
\begin{gathered}
T_{\mathbf{a}_{1}}: \psi e^{i \mathbf{K} \cdot \mathbf{r}_{i}} \rightarrow \psi e^{i \mathbf{K} \cdot\left(\mathbf{r}_{i}+\mathbf{a}_{1}\right)} \\
=\left(\psi e^{2 \pi i / 3}\right) e^{i \mathbf{K} \cdot \mathbf{r}_{i}} .
\end{gathered}
$$

Hence,

$$
T_{\mathbf{a}_{1}}: \psi \rightarrow \psi e^{2 \pi i / 3} .
$$

A translation by $\mathbf{a}_{2}$ acts the same way on $\psi$. A third example is $R_{x}$, the reflection $x \rightarrow-x$. Since $\mathbf{K}$ in Eq. (2.6) is parallel to $\hat{x}, R_{x}$ changes the sign of $\mathbf{K} \cdot \mathbf{r}_{\mathbf{j}}$, that is,

$$
\begin{gathered}
R_{x}: \operatorname{Re}\left[\psi e^{i \mathbf{K} \cdot \mathbf{r}_{i}}\right] \rightarrow \operatorname{Re}\left[\psi e^{-i \mathbf{K} \cdot \mathbf{r}_{i}}\right] \\
=\operatorname{Re}\left[\psi^{*} e^{i \mathbf{K} \cdot \mathbf{r}_{i}}\right] .
\end{gathered}
$$

Therefore,

$$
R_{x}: \psi \rightarrow \psi^{*} ;
$$

i.e., $R_{x}$ acts as complex conjugation. 
One can consider other symmetries of the hexagonal lattice, including rotations in the plane, the reflection $y \rightarrow-y$, and rotations by $180^{\circ}$ along an axis lying on the $z=0$ plane. However, in all cases the action of these transformations will reduce to one of the cases studied above, namely,

$$
\begin{gathered}
R_{z}: \psi \rightarrow-\psi, \\
T_{\mathbf{a}_{1}}: \psi \rightarrow \psi e^{2 \pi i / 3}, \\
R_{x}: \psi \rightarrow \psi^{*},
\end{gathered}
$$

or to combinations of these actions performed in sequence. Hence, it is sufficient to consider these three basic actions.

\section{Mapping to a six-state clock model}

After coarse graining the lattice, one can write down a continuum Landau free energy for the order parameter $\psi$, which must be invariant under all the underlying symmetries of the lattice. To sixth order in $\psi$ and $\psi^{*}$, and without derivatives, this restricts the allowed terms in the free energy to

$$
|\psi|^{2}, \quad|\psi|^{4}, \quad|\psi|^{6},
$$

as well as

$$
\frac{1}{2}\left[\psi^{6}+\left(\psi^{*}\right)^{6}\right]
$$

Note that the term $\frac{1}{2 i}\left[\psi^{6}-\left(\psi^{*}\right)^{6}\right]$ is forbidden since it is not invariant under $R_{x}$, Eq. (2.20). In addition, the lowest-order derivative term is

$$
|\nabla \psi|^{2}
$$

Thus, the Ginzburg-Landau (GL) free-energy density $f_{\mathrm{GL}}$ reads

$\frac{f_{\mathrm{GL}}}{\mathcal{K}}=\frac{\gamma}{2}|\nabla \psi|^{2}+r|\psi|^{2}+u|\psi|^{4}+v|\psi|^{6}+\frac{w}{2}\left[\psi^{6}+\left(\psi^{*}\right)^{6}\right]$,

which is scaled here by the Coulomb energy $\mathcal{K}$, Eq. (2.3). The coefficients in Eq. (2.24) can be obtained from the microscopic model of Eqs. (2.7) and (2.1) by expanding in small fluctuations starting from the high-symmetry disordered phase. We detail this expansion in Appendix A for the case of various power-law repulsions including the Coulomb interaction. For the specific case of the Coulomb interaction, the coefficient $\gamma$ is a number given by Eq. (A33). Using Eqs. (A14) and (A18), the coefficient of the quadratic term reads

$$
r=\frac{1}{\sqrt{3}}\left(\frac{C}{2 \mathcal{K}}-I_{2}\right)
$$

where $I_{n}$ is a constant whose explicit form is given in Eq. (A19). For the coefficient of the quartic term, Eq. (A23) yields

$$
u=\frac{3 \sqrt{3}}{4} I_{4} .
$$

With the help of Eq. (A26), we also find

$$
w=\frac{5}{8 \sqrt{3}} I_{6}
$$

and

$$
v=-\frac{25}{4 \sqrt{3}} I_{6} .
$$

Here, $I_{n}$ and $\gamma$ are constants explicitly given in Appendix A. The sign of $w$ determines the values of the phase $\theta$ that minimize the free energy. In particular, for $w>0$, this yields Eq. (2.10). We find that $v$ is negative, a situation that may result in a first-order phase transition. In Appendix A, it is checked that this is not the case. This behavior can be taken into account by adding a positive eighth-order term. Nevertheless, the correct physics can still be captured by Eq. (2.24) by setting $v>0$, as assumed for simplicity in the discussion of the next section.

When discussing quantum effects, we need to add dynamics. The lowest-order time-derivative term allowed by symmetry is

$$
\left|\partial_{t} \psi\right|^{2} .
$$

[Note that $i \psi^{*} \partial_{t} \psi$ is forbidden by Eq. (2.20)]. Hence, the effective time-dependent Ginzburg-Landau Lagrangian is

$$
\mathcal{L}_{\mathrm{GL}}=\frac{\gamma}{2 c^{2}}\left|\partial_{t} \psi\right|^{2}-f_{\mathrm{GL}},
$$

where

$$
c=\sqrt{\frac{\sqrt{3} \gamma \mathcal{K}}{2 M}}
$$

is the speed of sound for transverse (z-polarized) phonons.

\section{Critical point of the mean-field model}

In order to connect with previous results, we remark that our theory naturally delivers the critical value of the ratio $C / \mathcal{K}$ of the mean-field model, at which the planar crystal becomes unstable. This is found by setting $r=0$ in Eq. (2.25), and it yields the mean-field critical value 


$$
C_{c}^{\mathrm{MF}}=2 \mathcal{K} I_{2} \approx 13.36 \mathcal{K},
$$

which can be cast as a condition connecting the transverse trap frequency and the lattice constant:

$$
\omega_{z}^{\mathrm{MF}}=\left(2 I_{2} \frac{Q^{2} /\left(4 \pi \epsilon_{0}\right)}{M a^{3}}\right)^{1 / 2} .
$$

This value is consistent with that found in Ref. [32] (where it is reported in terms of the planar density $\sigma$ ). We further point out that $C_{c}^{\mathrm{MF}}$ is an upper bound to the critical value. As we will see below, at finite temperature, the planar instability is at a value $C_{c}(T)<C_{c}^{\mathrm{MF}}$ and decreases monotonically with $T$. At $T=0$, moreover, quantum fluctuations lower the value of the critical point from $C_{c}^{\mathrm{MF}}$ to $C_{c}(0)$ [so that $r_{c}<0$; see Eq. (2.25)] by a quantity involving the ratio of kinetic and potential energies, as shown in Ref. [53].

\section{E. Extension of the model to dipolar gases}

The mapping leading to the free-energy density in Eq. (2.24) can be performed by using, instead of ions, dipolar atoms or molecules that are polarized by an external field perpendicular to the plane $[51,52,54]$. In Appendix A 3 , we derive the coefficients of the Landau free-energy density, corresponding to Eq. (2.24), as a function of the dipolar interaction. It must be kept in mind, however, that the dipolar interaction is not sufficiently long range to warrant crystalline order in the plane, and the coupling with the planar modes can become relevant close to the meanfield critical point, changing the nature of the transition $[55,56]$. In principle, stability of the triangular lattice in the $x y$ plane can be enforced by a pinning potential, e.g., by an optical lattice. For this realization, the free energy in Eq. (2.24) describes the planar instability at small transverse displacements.

\section{PHASE DIAGRAM}

The mapping of the model to the Landau-Ginzburg free energy in Eq. (2.24) allows us to determine the phase diagram of the system as a function of the temperature $T$ and of the ratio $\mathcal{K} / C$, as depicted in Fig. 2. In ion Coulomb crystals, the first parameter is typically tuned by means of the laser that cools the ions, while the second ratio is controlled by changing the aspect ratio between the planar and the transverse trap frequencies. We discuss below the phase diagram for the transition at finite temperature and for the quantum phase transition at $T=0$.

\section{A. Thermal transitions}

Let us first consider the phase diagram at finite temperature. At the mean-field level, assuming $u, v, w>0$ and $v>w$, the free energy in Eq. (2.24) undergoes a secondorder phase transition when $r$ changes sign. For $r>0$, the system is disordered, $\psi=0$, whereas for $r<0$, the system is ordered in one of six minima of the free energy, $\psi=|\psi| e^{i \pi(2 n+1) / 6}$.

Beyond the mean field, in two spatial dimensions thermal fluctuations in the phase of the order parameter affect the phase diagram significantly. These fluctuations are captured by writing $\psi=\left|\psi_{0}\right| e^{i \theta}$, where $\left|\psi_{0}\right|$ is the mean-field value of the order parameter and $\theta$ is its phase. Then, Eq. (2.24) yields

$$
f_{\mathrm{GL}}=\frac{\rho_{s}^{0}}{2}(\nabla \theta)^{2}+h_{6} \cos (6 \theta),
$$

where $\rho_{s}^{0}$ is the bare superfluid stiffness,

$$
\rho_{s}^{0}=\gamma \frac{\mathcal{K}}{2}\left|\psi_{0}\right|^{2},
$$

and

$$
h_{6}=w\left|\psi_{0}\right|^{6}
$$

is a term that tends to $\operatorname{pin} \theta$ in one of the six values given by Eq. (2.10). On the level of mean-field theory for the model in Eq. (2.24), for $r<0$ the order parameter is different from zero and given by

$$
\left|\psi_{0}\right|^{2}=-\frac{r}{2 u}=\frac{1}{9}\left(\frac{2 I_{2}}{I_{4}}-\frac{C}{\mathcal{K} I_{4}}\right)
$$

(a similar expression is found for dipolar interactions-see Appendix A). The resulting values of the parameters in Eq. (3.1) are

$$
\rho_{s}^{0}=\gamma \frac{\mathcal{K}}{2} \frac{1}{9}\left(\frac{2 I_{2}}{I_{4}}-\frac{C}{\mathcal{K} I_{4}}\right)
$$

and

$$
h_{6}=\frac{5 \mathcal{K}}{8 \sqrt{3}} I_{6}\left[\frac{1}{9}\left(\frac{2 I_{2}}{I_{4}}-\frac{C}{\mathcal{K} I_{4}}\right)\right]^{3} .
$$

Equation (3.1) defines the six-state clock model, which has been shown to exhibit two separate phase transitions $[3,23]$. Let us consider $r$ fixed, $r<0$. Starting from high temperatures (but still below the crystallization temperature), the system is in a disordered phase in the transverse direction, with exponentially decaying correlations. Then, as the temperature is reduced below a critical temperature, which we denote by $T_{\mathrm{KT}}$, vortices bind and the system undergoes a Kosterlitz-Thouless (KT) transition $[1,2,8]$. The transition temperature is given by

$$
T_{\mathrm{KT}}=\pi \rho_{s} / 2,
$$

where the coefficient $\rho_{s}$ is the renormalized superfluid stiffness, which depends on $r$ and on temperature. It is smaller than the bare superfluid stiffness $\rho_{s}^{0}$ but is typically 
of the same order of magnitude, $\rho_{s} \lesssim \rho_{s}^{0}$. At the transition, the clock coupling $h_{6}$ is irrelevant, and an intermediate phase is reached in which the phase correlations are power law in nature,

$$
\left\langle e^{i \theta(\mathbf{r})} e^{-i \theta(0)}\right\rangle \sim|\mathbf{r}|^{-\eta}
$$

Here, $\eta$ is a continuous parameter, which is monotonically decreasing with temperature, and can be expressed as

$$
\eta=T /\left(2 \pi \rho_{s}\right) .
$$

It attains the universal value $\eta=1 / 4$ at $T_{\mathrm{KT}}$.

As the temperature is further reduced, when $\eta$ reaches the value $\eta=1 / 9$, i.e., at the temperature $T_{6}<T_{\mathrm{KT}}$, with

$$
T_{6}=\frac{2 \pi \rho_{s}}{9},
$$

the clock term becomes relevant and the system undergoes an additional transition. Below $T_{6}$, long-range $Z_{6}$ order is established, in which the phase of the order parameter is locked at one of the values in Eq. (2.10).

Let us now comment on the meaning of superfluid stiffness in the present context. While the crystal of ions is clearly not superfluid, this terminology is borrowed from superconductivity and is broadly applied to $X Y$ models. In this case, it is a helicity modulus, which measures the freeenergy increment associated with "twisting" the direction of the order parameter; see Eq. (3.1). Hence, it expresses the rigidity to deformations of the ordered pattern [57].

\section{B. Quantum critical point and its vicinity}

We next focus on the quantum phase transition at $T=0$. The temperature is a relevant variable at the quantum phase transition, and therefore, the transition is in a different universality class from the thermal transitions at $T>0$. Furthermore, in the region surrounding the quantum critical point, the finite-temperature phase diagram can be deduced from the scaling properties of the quantum critical point [58,59], as described below.

The quantum phase transition is described by the Lagrangian in Eq. (2.30). Using the quantum to classical correspondence [49], this can be mapped to a classical sixstate clock model, Eq. (3.1), but now living in three dimensions. The three-dimensional clock model has been found to have a direct continuous transition between the long-range ordered $Z_{6}$ phase and the disordered phase. The transition lies in the $X Y$ universality class [4-7], with the addition of the clock term $h_{6}$, which is found to be dangerously irrelevant at the critical point $[4,50]$. This implies that on both sides of the $T=0$ transition, the quantum problem has a critical energy scale that vanishes at the transition as

$$
\Delta \sim\left|r-r_{c}\right|^{\nu}
$$

where $\nu \approx 0.671$ is the correlation-length critical exponent of the $D=3 X Y$ transition [60]. In addition, when the transition is approached from the ordered phase, the dangerously irrelevant term $h_{6}$ introduces an additional critical energy scale, whose effects will be discussed at the end of this section.

In the region of the phase diagram where $h_{6}$ is irrelevant (i.e., in the disordered and the critical phases above $T_{6}$ in Fig. 2), $\Delta$ is the only critical energy scale. Hence, in these regions, scaling near the quantum critical point implies that the two-dimensional superfluid density behaves as [58]

$$
\rho_{s}(r, T)=T \Phi(\Delta / T),
$$

where $\Phi$ is a universal function of $\Delta / T$. In particular, this implies that the contours of constant exponent $\eta$ in Eq. (3.8) are also contours of constant $\Delta / T$. Hence,

$$
\begin{gathered}
T_{\mathrm{KT}}=A_{\mathrm{KT}}\left|r-r_{c}\right|^{\nu}, \\
T_{6}=A_{6}\left|r-r_{c}\right|^{\nu},
\end{gathered}
$$

where $A_{\mathrm{KT}}$ and $A_{6}$ are two nonuniversal constants. As the stiffness $\rho_{s}$ is monotonic in $T$ at fixed $\Delta$ (it is reduced by thermal fluctuations), and since $\eta$ is larger at $T_{\mathrm{KT}}$ than at $T_{6}$, it follows that $A_{\mathrm{KT}}>A_{6}$. Thus, we see that both transition temperatures go to zero at the quantum critical point, with the same exponent but different coefficients, and that the intermediate phase exists arbitrarily close to the quantum critical point, although it becomes narrow.

We finally note that, according to Ref. [5], within the ordered phase, there is a length scale $\xi_{6}$ larger than the $X Y$ correlation length $\xi$. In particular, near the quantum critical point, $\xi_{6}$ diverges faster, $\xi_{6} \sim \xi^{a_{6}}$, where $a_{6} \approx 9 / 4$. For distances smaller than $\xi_{6}$, the order parameter has effective $X Y$ symmetry, and only for distances beyond $\xi_{6}$ does the true long-range $Z_{6}$ order become apparent [5]. By the quantum to classical correspondence, this implies that in the $Z_{6}$ phase at $T=0$, there is a critical scale $\Delta_{6}$ that is softer than $\Delta, \Delta_{6} \sim \Delta^{a_{6}}$.

At first glance, one may expect the scaling of $T_{6}$ near the quantum critical point to be determined by $\Delta_{6}$ instead of $\Delta$. However, this is not the case since, when $T_{6}$ is approached from above, the relevance of the clock term is determined by the value of the superfluid stiffness alone. This implies Eq. (3.13), as outlined above. One may instead define a crossover temperature $T_{6}^{*}$, such that

$$
T_{6}^{*} \equiv \Delta_{6} \sim\left|r-r_{c}\right|^{a_{6} \nu} .
$$

Since $a_{6}>1$, the temperature $T_{6}^{*}$ is parametrically smaller than $T_{6}$ near the quantum critical point. For finite temperatures in the range $T_{6}^{*}<T<T_{6}$, the $Z_{6}$ ordering is 
characteristic of the classical $d=2$ model, while for $T<T_{6}^{*}$, it is dominated by the quantum $X Y$ model in $D=d+1=3$.

\section{Practical considerations for experimental realizations on ion traps}

We now make a number of quantitative estimates of parameters that may be useful in comparisons with experiments on ion traps. First, it is useful to connect the temperatures appearing in Sec. III A with experimental values for trapped ions. The features we predict will be observed below the crystallization temperature $T_{\text {cryst }}$, where $k_{B} T_{\text {cryst }} \simeq \mathcal{K} / \Gamma$ and $\Gamma$ is the plasma parameter at which crystallization occurs, $\Gamma \approx 140$ in two dimensions [61]. Equations (3.6) and (3.4) give $k_{B} T_{\mathrm{KT}} \simeq 0.011 \mathcal{K}\left(I_{2}-C / 2 \mathcal{K}\right)$. The temperature $T_{\mathrm{KT}}$ can thus be a fraction of the crystallization temperature depending on the distance of the ratio $C / \mathcal{K}$ from the mean-field critical value. For interparticle distances of the order of $15 \mu \mathrm{m}$, the crystallization temperature is in the millikelvin range [25]. For these parameters, sufficiently far away from the mean-field critical value, $T_{\mathrm{KT}}$ can be of the order of hundreds of $\mu$ Kelvin. Comparing Eqs. (3.6) and (3.9), the transition to long-range order is at a temperature $T_{6}$ of the same order as $T_{\mathrm{KT}}$.

These considerations suggest that the intermediate critical phase could be observed using sub-Doppler cooling techniques $[62,63]$. We remark that, in general, laser cooling can lead to different effective temperatures for the planar and transverse modes of the Coulomb crystal [37]. During the experiment, thermalization between the vibrations is typically not observed, which indicates that there is a sufficiently small coupling between axial and transverse vibrations, so that it can be neglected. This leads us to conjecture that corrections due to in-plane thermal fluctuations can also be neglected sufficiently close to the transition since in two dimensions the Coulomb interaction is sufficiently long-ranged to make the crystal incompressible along the plane [55]. This conjecture also corroborates the validity of our model, in which we assume that planar and transverse vibrations are consistently decoupled.

Next, we can estimate the size of quantum fluctuations in Sec. III B by computing the shift in quantum critical point $r_{c}$ that they induce. For this, we refer to Eq. (2.12) in Ref. [53] which, in the notation of the present paper, becomes

$$
r_{c}=-\frac{3^{5 / 4}}{\pi \gamma^{1 / 2}} u \tilde{\hbar}
$$

where $u$ is given by Eq. (2.26) while

$$
\tilde{\hbar}=\sqrt{\frac{\hbar^{2}}{2 M a^{2} \mathcal{K}}} .
$$

For $a=15 \mu \mathrm{m}$ and single charge ions, this results in

$$
r_{c}=-3.8 \times 10^{-4} \frac{1}{\sqrt{N_{A}}},
$$

where $N_{A}$ is the ion's atomic mass number. For beryllium, $N_{A}=9$, leading to

$$
r_{c}=-1.27 \times 10^{-4}
$$

This corresponds to a fractional shift in the trap frequency

$$
\frac{\delta \omega_{z}}{\omega_{z}}=\frac{\sqrt{3}}{2 I_{2}} r_{c}
$$

For a trap of frequency $1 \mathrm{MHz}$, the shift is $17 \mathrm{~Hz}$, and it could be measured when $\delta \omega_{z} T_{h} \ll 1$, with $T_{h}$ the heating time scale of the ion trap $[37,64]$.

The shift in $r_{c}$ in Eq. (3.18) can also be compared with the width of the buckled phase. Based on the stability analysis of Ref. [32], the three-layer phase is expected to exist over a range of planar densities $\sigma$, given by $\sigma a_{0}^{2} \in[1.11,1.15]$, where $a_{0}$ is a length scale extracted from the trapping potential. This somewhat narrow range is limited at high densities by a first-order transition into a staggered square lattice structure, which occurs at $\sigma=1.15 / a_{0}^{2}$. In terms of the parameter $r$, the lower density corresponds to $r=0$, where the mean-field onset of the three layers occurs, whereas the higher density corresponds to

$$
r^{(\text {first order })}=-\frac{\sqrt{3} I_{2}}{2} \frac{\delta \sigma}{\sigma}=-0.21,
$$

where $\delta \sigma / \sigma=(1.15-1.11) / 1.11$ is the relative width of the phase. Hence, the fluctuation-induced shift in Eq. (3.18) is very small relative to the full width of the phase.

Another practical issue to address is the effect of the finite system size. For harmonic confinement, the triangular crystalline structure can be observed with 1000 ions at the center of the plane [26]. Present experiments can realize planes with about 1000 ions, corresponding to a linear dimension of $L \approx 30$ ions. Deep in the ordered and disordered phases, when the correlation length $\xi$ is well below $L$, the finite size of the system is not important. By contrast, as the critical phase is approached within either of these phases, $\xi$ diverges and the finite system size must be taken into account. In particular, when $\xi$ is of order $L$, the system will appear to be critical. This places a practical limitation in mapping out the phase transition lines. However, by studying the dependence on system size, it is possible to locate these transitions with high accuracy using relatively modest system sizes. For example, in Ref. [65], the Kosterlitz-Thouless temperature was 
determined with an accuracy of three significant digits by simulating systems of size $12 \times 12$ and smaller.

On the other hand, within the critical phase itself, the finite system size does limit the ability to accurately measure critical exponents since $L=30$ gives access to only a relatively short segment of a power law. A rough rule of thumb is that one can extract one significant digit for each decade of scaling, so here we may expect to obtain the first digit of the critical exponents. Finally, the observation of the scaling form for $T_{6}^{*}$, given in Eq. (3.14), will require larger system sizes than 1000 atoms. Close to the quantum critical point, in fact, this crossover occurs at a long correlation length, which is associated with the dangerously irrelevant clock term. Far from the critical point, $T_{6}^{*}$ might be extracted from the temperature dependence of the clock order parameter, as discussed below.

\section{MEASURABLE SIGNATURES OF THE PHASE DIAGRAM}

We now focus on physical quantities that are, in principle, accessible to experimental probes. The mechanical instability of the two-dimensional Coulomb crystal gives rise to ordering in the transverse direction, which occurs in two stages: For temperatures $T$ between $T_{6}$ and $T_{\mathrm{KT}}$, there is a critical phase with quasi-long-range order [3]. For $T<T_{6}$, in the ordered phase, the system spontaneously selects one of six equivalent patterns, i.e., breaks $Z_{6}$ symmetry. This structural phase transition is therefore a realization of the ordering in a six-state clock model, manifested here by splitting of the single layer of ions into three layers, at $z=0, \pm h$. Hence, the layer separation $h$ serves as a measurable probe of the order parameter. In addition, the resulting enlargement of the unit cell in the ordered pattern of Fig. 1 is expected to induce new Bragg peaks in a diffraction experiment. Their height and line shape as functions of $T$ and the system parameters can provide a further test of the predictions of the theoretical model. In this section, we also propose a possible observable for the classical-quantum crossover within the ordered phase at a characteristic temperature $T_{6}^{*}$. For temperatures below $T_{6}^{*}$, the layer separation $h(T)$ is expected to saturate to a constant value. This criterion will serve as an operational definition of $T_{6}^{*}$.

The discussion of this section is centered on determining the signatures of the phase diagram by direct measurement of the crystal planes and by a Bragg diffraction experiment. Bragg scattering could be performed at the asymptotics of laser cooling, when the ions have reached the stationary state and the emitted photons carry the information about the crystal structure and excitations [35,66]. In addition, it is worth mentioning that the transverse shift of the crystal planes could also be measured by mapping the crystal structure into the electronic degrees of freedom of the ions [37,67-69]. These methods can, in principle, provide precise information of the transverse structure and fluctuations. Moreover, thanks to the advances in high-precision spectroscopy, such measurements can provide the precision required to access the quantum regime.

\section{A. Temperature dependence of the layer separation}

Assuming that direct imaging of the particles is possible, the ordering established in the dark shaded area of Fig. 2 would be manifested as splitting of the plane to three layers separated by a distance

$$
h=\max _{i}\left(\left|\left\langle z_{i}\right\rangle\right|\right),
$$

where $z_{i}$ is given by Eq. (2.8) with $i$ on the + or - sublattice of the broken-symmetry pattern (Fig. 1), and $\langle\ldots\rangle$ denotes the thermal expectation value. Expressing the phase of the order parameter field $\psi$ as $\theta=\theta_{0}+\delta \theta$, where $\theta_{0}$ is one of the clock states $(\pi / 6)(2 n+1)$, one obtains

$$
h=\left|\psi_{0}\right| \cos (\pi / 6)\langle\cos (\delta \theta)\rangle,
$$

where $\psi_{0}$ is given in Eq. (3.3). While in the intermediate critical phase the ions exhibit thermal fluctuations between the three layers, for $r<r_{c}$ and $T<T_{6}$ the expectation value $\langle\cos (\delta \theta)\rangle \neq 0$, and consequently $h$ is finite in the thermodynamic limit. It can be evaluated using an effective Gaussian theory for the phase fluctuations $\delta \theta$ (see Appendix B for details). This yields

$$
\left.h(T)\right|_{r<r_{c}, T<T_{6}}=h_{0}\left[2 \sinh \left(\frac{m(T)}{2 T}\right)\right]^{[\eta(T) / 2]} ;
$$

here, $m(T)$ is a temperature-dependent coefficient, whose explicit form is given in Appendix B, and

$$
h_{0}=\left|\psi_{0}\right| \cos (\pi / 6) e^{-\left(c \Lambda / 8 \pi \rho_{s}\right)}
$$

is a nonuniversal amplitude that depends on the highmomentum cutoff $\Lambda \approx \pi / a$. In the quantum regime, i.e., for $T \ll T_{6}^{*}$ (below the dashed line in Fig. 2), Eq. (4.3) can be approximated by the temperature-independent value

$$
\left.h(T)\right|_{T \ll T_{6}^{*}}=h_{0} e^{\left(m_{0} / 8 \pi \rho_{s}\right)},
$$

where $m_{0}$ is given in Eq. (B18) of Appendix B. In fact, the saturation itself of $h(T)$ occurs at $T \approx m_{0}$, which according to our definition, allows us to identify

$$
T_{6}^{*} \approx m_{0} .
$$

The saturation value, Eq. (4.5), becomes invalid close to the quantum critical point $\left(r \rightarrow r_{c}, T=0\right)$, where

$$
\left.h\right|_{r \rightarrow r_{c}, T=0} \sim\left|r-r_{c}\right|^{\beta},
$$


with $\beta \approx 0.348$ the $X Y$ critical exponent $[5,60]$ at $D=3$. In the classical regime $\left(T_{6}^{*} \ll T<T_{6}\right)$,

$$
\begin{aligned}
\left.h(T)\right|_{T_{6}^{*} \ll T<T_{6}} & =h_{0}\left(\frac{m(T)}{T}\right)^{[\eta(T) / 2]} \\
& \approx h_{0}\left(\frac{m_{0}}{T}\right)^{\left\{T /\left[18\left(T_{6}-T\right)\right]\right\}},
\end{aligned}
$$

where in the final expression, we employed Eq. (B19) of Appendix B and also Eqs. (3.8) and (3.9).

In order to illustrate the temperature dependence of $h(T)$, we consider beryllium ions with lattice constant $a=15 \mu \mathrm{m}$. Then, combining Eqs. (2.25), (3.4), (3.5), and (B18), we obtain

$$
\begin{gathered}
h_{0}=(4.3 \mu \mathrm{m}) \times \sqrt{|r|}, \\
m_{0}=\left(1.3 \times 10^{-5} \text { Kelvin }\right) \times|r| .
\end{gathered}
$$

These expressions are mean field in nature and do not take fluctuations into account, but they do give rough estimates for the values of the parameters. In particular, since $r$ is bounded by Eq. (3.20), $|r|<0.21, m_{0}$ is in the $\mu \mathrm{K}$ range (by comparison, for similar parameters, $\rho_{s}$ is of order a few $\mathrm{mK})$. This value is small compared to typical experimental temperatures. Hence, Eq. (4.9) provides a good approximation to the order parameter $h(T)$. Figure 3 shows the temperature dependence of $h(T)$ for a number of values of $m_{0}$. Note that although $m_{0}$ of this magnitude is too small to be accessed directly in experiments, its value affects the temperature dependence of $h(T)$ at $T \gg m_{0}$. Hence, it may be possible to extract an estimate for $m_{0}$ (and therefore of $T_{6}^{*}$ ) from a fit to $h(T)$, using Eq. (4.9).

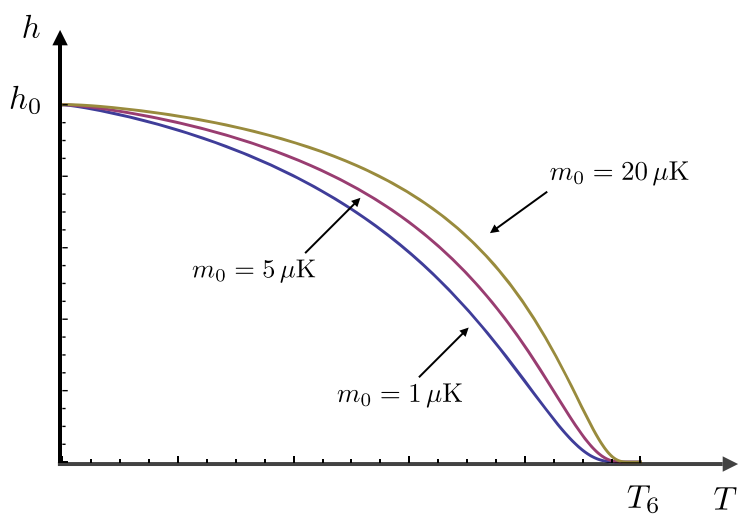

FIG. 3. Interlayer separation $h(T)$ as a function of temperature, based on Eq. (4.9). The zero-temperature value, $h_{0}$, is of order 1 micron, as shown in Eq. (4.4). Note that the functional form of $h(T)$ is sensitive to the value of $m_{0}$, even at temperatures well above $m_{0}$ itself. Throughout, the onset temperature for clock order is taken to be $T_{6}=10 \mathrm{mK}$.

\section{B. Diffraction pattern}

Another measurable quantity is the diffraction pattern $I_{\mathbf{k}_{3 d}}$ at wave vector $\mathbf{k}_{3 d}=\left(\mathbf{k}, k_{z}\right)$, where $\mathbf{k}$ is a vector in the $x y$ plane. The diffraction pattern probes the structure factor $S_{\mathbf{k}_{3 d}}$, that is, the Fourier transform of the density operator, via the quantity

$$
I_{\mathbf{k}_{3 d}}=\left\langle\left|S_{\mathbf{k}_{3 d}}\right|^{2}\right\rangle .
$$

For $k_{z} h \ll 1$, this expression can be cast in the form

$$
I_{\mathbf{k}_{3 d}}=I_{\left(\mathbf{k}, k_{z}=0\right)}+F\left(\mathbf{k}, k_{z}\right),
$$

with the leading $k_{z}$-dependent contribution

$$
\begin{aligned}
F\left(\mathbf{k}, k_{z}\right) & =\frac{k_{z}^{2}\left|\psi_{0}\right|^{2} N}{4} \sum_{j}\left(e^{i(\mathbf{k}+\mathbf{K}) \cdot \mathbf{r}_{\mathbf{j}}}+e^{i(\mathbf{k}-\mathbf{K}) \cdot \mathbf{r}_{\mathbf{j}}}\right) C\left(\mathbf{r}_{\mathbf{j}}\right) \\
C(\mathbf{r}) & \equiv\left\langle e^{i(\delta \theta(\mathbf{r})-\delta \theta(0))}\right\rangle
\end{aligned}
$$

(details are given in Appendix C). This contribution exhibits peaks at new wave vectors $\mathbf{k}_{0}= \pm \mathbf{K}+\mathbf{G}$, where $\mathbf{K}$ is given by Eq. (2.6) and $\mathbf{G}$ is a reciprocal lattice vector, reflecting the enlarged unit cell. Defining the small deviation $\mathbf{q}=\mathbf{k}-\mathbf{k}_{0}$, we get

$$
F\left(\mathbf{q}, k_{z}\right) \approx \frac{k_{z}^{2}\left|\psi_{0}\right|^{2} N}{4} \int d^{2} r e^{i \mathbf{q} \cdot \mathbf{r}} C(\mathbf{r}) .
$$

Within the Gaussian effective theory [Eq. (C6) in Appendix C], the asymptotic form of $C(\mathbf{r})$ for large $\mathbf{r}$ is

$$
\begin{aligned}
C(\mathbf{r}) & \approx\langle\cos (\delta \theta)\rangle^{2}(1+\langle\delta \theta(\mathbf{r}) \delta \theta(0)\rangle) \\
& =\mathrm{const}+\delta C(\mathbf{r}),
\end{aligned}
$$

where in the entire ordered phase $\left(T<T_{6}, r<r_{c}\right)$, the correlation function $\delta C(\mathbf{r})$ is exponentially decaying over a length $c / m$ [see Eq. (C8)]. Consequently, the line shape of the new diffraction peaks $\left[F\left(\mathbf{q}, k_{z}\right)\right.$ defined in Eq. (4.15)] will be given by $\delta(\mathbf{q})+F_{\text {fluc }}(\mathbf{q})$, where the fluctuation contribution $F_{\text {fluc }}(\mathbf{q})$ has the form of a Lorentzian with a characteristic width $\sim m(T) / c$.

We finally focus on the critical phase established in the higher- $T$ regime $T_{6}<T<T_{\mathrm{KT}}$ (the light-shaded region in Fig. 2), where quasi-long-range order is anticipated. The hallmark of such a phase is a critical behavior of the correlation functions $C(\mathbf{r})$, which should manifest the power-law decay of Eq. (3.7) with the $T$-dependent exponent $\eta$. As $T$ is reduced from the upper critical temperature $T_{\mathrm{KT}}$, this exponent varies continuously from $\eta=1 / 4$ at $T_{\mathrm{KT}}$ to $\eta=1 / 9$ at $T_{6}$. We expect that this behavior will be reflected by the appearance of smeared Bragg peaks at the new wave vectors $\mathbf{k}_{0}$. Their line shape $F\left(\mathbf{q}, k_{z}\right)$ should exhibit the power-law dependence [11] 


$$
F\left(\mathbf{q}, k_{z}\right) \sim|\mathbf{q}|^{\eta-2} .
$$

Hence, in principle, a diffraction measurement can serve as a direct probe of the exponent $\eta(T)$.

\section{CONCLUSIONS}

In conclusion, we have argued that the mechanical instability of a planar crystal of trapped ions can be mapped to the six-state clock model and thus manifests a two-step buckling transition. On the basis of symmetry consideration, we derived the field-theoretical model and determined the coefficients starting from the microscopic Hamiltonian. The phase diagram is characterized by several phases, depicted in Fig. 2 as a function of the temperature $T$ and of the external trap frequency, here parametrized by the coefficient $r$. It exhibits an intermediate, critical phase between disorder and long-range order in the buckled pattern of transverse displacements, within a finite range of parameters. This intermediate phase shrinks to zero as $T \rightarrow 0$, where a unique critical point is expected. Finally, we argued that within the ordered phase, a crossover from a classical to a quantum behavior occurs at a yet-lower temperature $T_{6}^{*}(r)<T_{6}$. This signifies the emergence of an additional characteristic scale for clock order, $\xi_{6} \sim 1 / T_{6}^{*}$, which is intimately related to the clock term being dangerously irrelevant.

These behaviors are manifested in the functional dependence of the interlayer distance $h$ and of the Bragg signal, accessible in experiments with trapped ions, on the trap frequency and on the temperature of the crystal. In particular, in a pancake-shaped trap of the type used in Ref. [36], a gradual change of particle density towards the center of the $x y$ plane can yield a "wedding cake" structure [70] and would enable the probing of $h$ vs the tuning parameter $r$ as it gradually varies with the distance from the center.

We finally note that according to our estimate of the parameters for ion crystal systems, the thermal transition lines $T_{\mathrm{KT}}(r)$ and $T_{6}(r)$ are expected to be observable with present trapping and cooling techniques. Direct probing of the quantum regime (by cooling to temperatures of order $T_{6}^{*}$ ) is more challenging. However, information on quantum effects can be drawn from measurements done at accessible, higher temperatures $\left(T \gg T_{6}^{*}\right)$. For example, by fitting the curve $h$ vs $T$ to the theory, the energy scale $T_{6}^{*}$ can be extracted. In addition, by analyzing the critical temperatures $T_{\mathrm{KT}}, T_{6}$ as a function of $r$, it may be possible to verify the critical exponent for the quantum transition [see Eqs. (3.12) and (3.13)], even far from the quantum critical point. Furthermore, the enhanced quantum fluctuations in systems of ultracold dipolar gases, such as in the setup of Ref. [54], provide an independent realization in which quantum effects may be observed and measured more directly.

\section{ACKNOWLEDGMENTS}

We are grateful to Joe Britton, John Bollinger, Alexey Gorshkov, Shlomi Kotler, Mikhail Lukin, Yoav Sagi, and especially to Eugene Demler for illuminating discussions. This work was partially supported by the German Research Foundation (DFG) project DACH: Quantum Crystals of Matter and Light; the Israel Science Foundation (ISF) Grants No. 1839/13, No. 231/14, and No. 1028/12; the Joint UGC-ISF Research Grant Program under Grant No. 1903/14; the US-Israel Binational Science Foundation (BSF) Grant No. 2010132; and by the Shlomo Kaplansky academic chair. E. S., G. M., and S. F. thank the Kavli Institute for Theoretical Physics in Santa Barbara for its hospitality, where this research was supported in part by the National Science Foundation (NSF) under Grant No. NSF PHY1125915. D. P. and E. S. thank the Aspen Center for Physics, where part of this work was done, with support of NSF Grant No. PHY-1066293, and for the support of the Simons Foundation. G. M. thanks the Ion Storage Group at NIST, Boulder, for hospitality and support during completion of this work.

\section{APPENDIX A: DERIVATION OF THE GL FREE ENERGY}

Long-range order results in the increase of the unit cell; therefore, we turn first to find the wave vectors of longrange order. Since the interaction is the strongest for nearest neighbors, we assume the nature of the long-range order is determined by the nearest neighbors, while further neighbors determine the numerical values of parameters. This assumption is tested numerically.

\section{The order determined by nearest-neighbor interactions}

The potential energy for nearest-neighbor interactions can be written after truncating the Coulomb sum, and it takes the form

$$
V=\frac{C}{2} \sum_{i} z_{i}^{2}-\frac{\mathcal{K}}{4} \sum_{\langle i, j\rangle}\left(z_{i}-z_{j}\right)^{2},
$$

where $\langle i, j\rangle$ denotes the sum over nearest neighbors. Since the long-range order is characterized by a wave vector, it is convenient to introduce the Fourier expansion of the transverse displacement,

$$
z_{i}=\sum_{\mathbf{k}} \tilde{z}_{\mathbf{k}} e^{i \mathbf{k} \cdot \mathbf{r}_{i}}
$$

where $\mathbf{r}_{i}$ is a point on the two-dimensional lattice and $z_{i}$ is the distance of the ion at this point from the plane. In Fourier space, the first term in Eq. (A1) reads

$$
V_{1}=\frac{C}{2} \sum_{i} z_{i}^{2}=\frac{C}{2} \sum_{\mathbf{k}}\left|\tilde{z}_{\mathbf{k}}\right|^{2},
$$


where we have used that $z_{-\mathbf{k}}=z_{\mathbf{k}}^{*}$, with $z_{i}$ being real. The second term on the right-hand side of Eq. (A1) is given by

$$
V_{2}=\mathcal{K} \sum_{\mathbf{k}}\left|\tilde{z}_{\mathbf{k}}\right|^{2} F(\mathbf{k})
$$

with

$$
F(\mathbf{k})=\sum_{i=1}^{6} \sin ^{2}\left(\frac{1}{2} \mathbf{k} \cdot \delta \mathbf{r}_{i}\right) .
$$

Here, $\delta \mathbf{r}_{i}$ are the vectors that join some site of the planar lattice to the corresponding six nearest neighbors; they are combinations of $\mathbf{a}_{1,2}$ with integer coefficients $\pm 1,0$. Using their explicit form,

$$
F(\mathbf{k})=3-\cos k_{x}-2 \cos \frac{1}{2} k_{x} \cos \frac{\sqrt{3}}{2} k_{y} .
$$

Finally, in Fourier space, potential (A1) takes the form

$$
V=V_{1}-V_{2}=\sum_{\mathbf{k}}\left|\tilde{z}_{\mathbf{k}}\right|^{2}\left(\frac{C}{2}-\mathcal{K} F(\mathbf{k})\right) .
$$

For sufficiently large confinement $C$, the ions are confined to the $x y$ plane. This is the high-symmetry disordered phase. According to Landau theory, long-range order is expected for a vector $\mathbf{k}=\left(k_{x}, k_{y}\right)$ for which the sign of the corresponding term in the sum (A7) first changes sign as $C$ is increased. This happens for the value of $\mathbf{k}$ that maximizes $F(\mathbf{k})$. A straightforward calculation shows that the appropriate wave vectors are the corners of the Brillouin zone $\pm \mathbf{K}$, where $\mathbf{K}$ is given by Eq. (2.6), namely,

$$
\mathbf{K}=\left(\frac{4 \pi}{3}, 0\right),
$$

as well as for other vectors related to them by the reciprocal lattice vectors. In particular, for $\mathbf{K}$ in Eq. (A8), $F(\mathbf{K})=\frac{9}{2}$, and in the vicinity of the critical point, the energy related to the long-range order reads

$$
U_{\mathbf{K}}=\left(\frac{C}{2}-\frac{9}{2} \mathcal{K}\right)\left|z_{\mathbf{K}}\right|^{2}
$$

The contribution of the nearest neighbors to the gradient term in the GL free-energy density is

$$
V_{f}^{\prime}=\frac{3}{4} \mathcal{K}|\delta \mathbf{k}|^{2}\left|\tilde{z}_{\mathbf{K}}\right|^{2},
$$

where $\delta \mathbf{k}$ is the deviation from $K$, or

$$
V_{f}^{\prime}=\frac{3}{8} \mathcal{K}|\nabla \psi|^{2}
$$

and which thus delivers the value of the constant $\gamma$ in Eq. (2.24) for nearest-neighbor interactions. In the following, the contribution of farther neighbors will be calculated.

\section{Parameters of the GL free energy for the Coulomb potential}

We now derive the parameters of the Ginzburg-Landau free energy, Eq. (2.24), taking the full Coulomb interaction of Eq. (2.7). Using the order parameter (2.8), the distance from the $x y$ plane takes the form

$$
z_{i}=|\psi| \cos \left(\mathbf{K} \cdot \mathbf{r}_{i}+\theta\right),
$$

where

$\mathbf{r}_{i} \equiv \mathbf{r}\left(n_{1, i}, n_{2, i}\right)=\left(\frac{1}{2}\left(n_{1, i}+n_{2, i}\right), \frac{\sqrt{3}}{2}\left(n_{1, i}-n_{2, i}\right)\right)$,

with $n_{1, i}, n_{2, i}=0, \pm 1, \pm 2, \ldots$, and $\mathbf{K}$ is given in Eq. (2.6). Substituting Eq. (A12) into Eq. (2.1), the contribution of the harmonic potential to the GL free energy is written as

$$
U_{c}=N \frac{C}{4}|\psi|^{2}=\Omega \frac{C}{2 \sqrt{3}}|\psi|^{2},
$$

where $N$ is the number of ions and

$$
\Omega=\frac{\sqrt{3}}{2} N
$$

is the area of the sample (in units of $a^{2}$ ). In order to obtain the GL free energy of Eq. (2.24), we expand the Coulomb energy $U_{Q}$, Eq. (2.7), in powers of $z_{i}$. Since the plane is an equilibrium configuration, the first-order term vanishes. The second-order term reads

$$
U_{2, Q}=-\frac{\mathcal{K}}{4} \sum_{i, j} \frac{\left(z_{i}-z_{j}\right)^{2}}{\left|\mathbf{r}_{i}-\mathbf{r}_{j}\right|^{3}},
$$

and in terms of the order parameter, $\psi$ of Eq. (2.8) can be recast in the form

$$
\begin{aligned}
\left(z_{i}-z_{j}\right)^{2}= & 4|\psi|^{2} \sin ^{2}\left(\frac{\mathbf{K} \cdot\left(\mathbf{r}_{j}-\mathbf{r}_{i}\right)}{2}\right) \\
& \times \sin ^{2}\left(\frac{\mathbf{K} \cdot\left(\mathbf{r}_{j}-\mathbf{r}_{i}\right)}{2}+\mathbf{K} \cdot \mathbf{r}_{i}+\theta\right) .
\end{aligned}
$$

Performing the sum in Eq. (A16) over $i$ for fixed $\left(\mathbf{r}_{j}-\mathbf{r}_{i}\right)$ [which can be considered a lattice vector of the form (A13)], we find 


$$
U_{2, Q}=-\frac{\Omega \mathcal{K}}{\sqrt{3}}|\psi|^{2} I_{2},
$$

where

$$
I_{n}=\sum_{n_{1}, n_{2}} \frac{\sin ^{n}\left[\frac{\pi}{3}\left(n_{1}+n_{2}\right)\right]}{\left|\mathbf{r}_{n_{1}, n_{2}}\right|^{n+1}},
$$

with

$$
\left|\mathbf{r}_{n_{1}, n_{2}}\right|=\sqrt{\frac{1}{4}\left(n_{1}+n_{2}\right)^{2}+\frac{3}{4}\left(n_{1}-n_{2}\right)^{2}} .
$$

The sum is over all integers $n_{1}$ and $n_{2}$, excluding the point $n_{1}=n_{2}=0$. This sum can be calculated numerically, and one finds $I_{2}=6.6830$, where the contribution of nearest neighbors is $\frac{9}{2}$. Combining the contributions (A14) and (A18), one finds

$$
r=\frac{1}{\sqrt{3}}\left(\frac{C}{2 \mathcal{K}}-I_{2}\right),
$$

where we rescaled the term by $\mathcal{K}$, as in Eq. (2.24). Note that $U_{2, Q}$ does not depend on $\theta$, as expected from the general symmetry considerations. This is also the property of the coefficient for the quartic term, as we show now. The fourth-order contribution to the Coulomb energy is

$$
U_{4, Q}=\frac{3 \mathcal{K}}{16} \sum_{i, j} \frac{\left(z_{i}-z_{j}\right)^{4}}{\left|\mathbf{r}_{i}-\mathbf{r}_{j}\right|^{5}}
$$

Using similar manipulations as for the second order, one finds

$$
U_{4, Q}=\Omega \frac{9 \mathcal{K}}{4 \sqrt{3}}|\psi|^{4} I_{4},
$$

with $I_{4}=3.5596$. After rescaling by $\mathcal{K}$, this leads to the coefficient

$$
u=\frac{3 \sqrt{3}}{4} I_{4}
$$

The sixth-order contribution to the Coulomb energy is

$$
U_{6, Q}=-\frac{5 \mathcal{K}}{32} \sum_{i, j} \frac{\left(z_{i}-z_{j}\right)^{6}}{\left|\mathbf{r}_{i}-\mathbf{r}_{j}\right|^{7}}
$$

Using similar manipulations, again one finds

$$
U_{6, Q}=\Omega \frac{5 \mathcal{K}}{8 \sqrt{3}}|\psi|^{6}[\cos (6 \theta)-10] I_{6} .
$$

This is the nonvanishing term at lowest order that depends on $\theta$, as expected from symmetry. From Eq. (A26), we identify the coefficients

$$
w=\frac{5}{8 \sqrt{3}} I_{6}
$$

and

$$
v=-\frac{25}{4 \sqrt{3}} I_{6},
$$

where numerical evaluation yields $I_{6}=2.5577$.

Note that $v$ is negative. This may indicate a first-order phase transition. However, we have found that this is not the case by numerical evaluation of the energy at fixed wave vector $\mathbf{K}$, as a function of the order parameter $\psi$. For a large confining potential, above the critical confining potential, $C_{c}=2 \mathcal{K} I_{2}$, the minimum energy lies at $\psi=0$. At $C_{c}$, the minimum is still at $\psi=0$, and energy is quartic in $\psi$ at small $\psi$. Below $C_{c}$, the minimum energy shifts away from $\psi=0$ and evolves smoothly as a function of $C$. Hence, there is no first-order phase transition, at least at the mean-field level, despite the negative value of $v$. This results from positive higher-order terms, such as $|\psi|^{8}$, which stabilize the second-order phase transition.

In order to calculate the gradient term of Eq. (2.24), taking the contribution of all neighbors into account, we consider the second-order term of the expansion in the Coulomb interaction for deviation of the wave number $\delta \mathbf{k}$ from $\mathbf{K}$ :

$$
U_{2, Q}(\delta \mathbf{k})=-\frac{N \mathcal{K}}{2} \sum_{n_{1}, n_{2}} \frac{\sin ^{2}\left(\frac{1}{2}(\mathbf{K}+\delta \mathbf{k}) \cdot \mathbf{r}_{n_{1}, n_{2}}\right)}{\left|\mathbf{r}_{n_{1}, n_{2}}\right|^{3}}|\psi|^{2},
$$

where $\mathbf{r}_{n_{1}, n_{2}}$ is given by Eq. (A13). Expanding in $\delta \mathbf{k}$, the zeroth-order term yields Eq. (A18), whereas the first-order term vanishes in the sum. The second-order term is

$$
\delta U_{2, Q}=-\frac{N \mathcal{K}}{2} \sum_{n_{1}, n_{2}} \frac{\cos \left(\mathbf{K} \cdot \mathbf{r}_{n_{1}, n_{2}}\right)\left(\delta \mathbf{k} \cdot \mathbf{r}_{n_{1}, n_{2}}\right)^{2}}{4\left|\mathbf{r}_{n_{1}, n_{2}}\right|^{3}}|\psi|^{2},
$$

which has the form $\delta U_{2, Q}=M_{a b} \delta k_{a} \delta k_{b}$, where the coefficient matrix $M_{a b}$ is symmetric and real. At the $\mathbf{K}$ point, the system is invariant under $120^{\circ}$ rotations. This implies that the eigenvalues of $M_{a b}$ must be degenerate; i.e., $M_{a b}$ must be proportional to the $2 \times 2$ identity matrix. Otherwise, the eigenvectors of $M_{a b}$ would pick special directions in the $x y$ plane, thus breaking the rotational symmetry by $120^{\circ}$. Hence, $M_{x y}=0$ and $M_{x x}=M_{y y}$, allowing us to write $M_{a b}=\frac{M_{x x}+M_{y y}}{2} \delta_{a b}$. This gives

$$
\delta U_{2, Q}=\frac{\Omega \mathcal{K}}{2} \gamma|\delta \mathbf{k}|^{2}|\psi|^{2},
$$


with

$$
\begin{aligned}
\gamma & =-\sum_{n_{1}, n_{2}} \frac{\cos \left[\mathbf{K} \cdot \mathbf{r}_{n_{1}, n_{2}}\right]}{4 \sqrt{3}\left|\mathbf{r}_{n_{1}, n_{2}}\right|} \\
& =0.226 .
\end{aligned}
$$

The resulting gradient term is

$$
U_{\nabla, Q}=\gamma \frac{\Omega \mathcal{K}}{2}|\nabla \psi|^{2},
$$

which determines the coefficient $\gamma$ in Eq. (2.24).

\section{Parameters of the GL free energy for the dipolar potential}

We now calculate the parameters of the GinzburgLandau free energy for dipolar interactions. Let us assume dipoles of magnitude $P$ are oriented by an external field to point in the $z$ direction. The interaction energy for a pair of dipoles connected by a vector $\mathbf{r}$ is in units of the lattice spacing,

$$
U^{\prime}=\frac{\mathcal{K}_{D}}{|\mathbf{r}|^{3}}\left[1-3(\hat{\mathbf{z}} \cdot \hat{\mathbf{r}})^{2}\right],
$$

where

$$
\mathcal{K}_{D}=\frac{P^{2}}{a^{3}}
$$

while $\hat{\mathbf{z}}$ and $\hat{\mathbf{z}}$ are unit vectors in the $z$ and $r$ directions, respectively. For dipoles in the $x y$ plane, then $(\hat{\mathbf{z}} \cdot \hat{\mathbf{r}})=0$. The energy resulting from the displacement of dipoles from the $x y$ plane is

$$
\begin{aligned}
U_{D}= & \frac{\mathcal{K}_{D}}{2} \sum_{i \neq j} \frac{\left(x_{i}-x_{j}\right)^{2}+\left(y_{i}-y_{j}\right)^{2}-2\left(z_{i}-z_{j}\right)^{2}}{\left|\left(x_{i}-x_{j}\right)^{2}+\left(y_{i}-y_{j}\right)^{2}+\left(z_{i}-z_{j}\right)^{2}\right|^{5 / 2}} \\
& -\frac{\mathcal{K}}{2} \sum_{i \neq j} \frac{1}{\left|\left(x_{i}-x_{j}\right)^{2}+\left(y_{i}-y_{j}\right)^{2}\right|^{3 / 2}} .
\end{aligned}
$$

The vector $\mathbf{K}$ of long-range order is determined by the nearest neighbors, as in the Coulomb case, and takes the values of Eqs. (2.6) and (A8). In what follows, we expand $U_{D}$ in powers of $z_{i}$ and use similar manipulations as in the Coulomb case. The second-order term is

$$
U_{2, D}=-\frac{9 \mathcal{K}_{D}}{4} \sum_{i, j} \frac{\left(z_{i}-z_{j}\right)^{2}}{\left|\mathbf{r}_{i}-\mathbf{r}_{j}\right|^{5}} .
$$

Writing the expression in terms of the order parameter $\psi$ of Eq. (2.8) by performing a calculation similar to the one resulting in Eq. (A18), we find

$$
U_{2, D}=-\Omega \frac{9 \mathcal{K}_{D}}{\sqrt{3}}|\psi|^{2} I_{2}^{D}
$$

where

$$
I_{n}^{D}=\sum_{n_{1}, n_{2}} \frac{\sin ^{n}\left[\frac{\pi}{3}\left(n_{1}+n_{2}\right)\right]}{\left|\mathbf{r}_{n_{1}, n_{2}}\right|^{n+3}}
$$

with $\left|\mathbf{r}_{n_{1}, n_{2}}\right|$ given by Eq. (A20). The sum is over all integers $n_{1}$ and $n_{2}$, excluding the point $n_{1}=n_{2}=0$. This sum can be calculated numerically, and one finds $I_{2}^{D}=-4.746$. Combining the contributions (A14) and (A39), one finds

$$
r=\frac{1}{\sqrt{3}}\left(\frac{C}{2 \mathcal{K}_{D}}-9 I_{2}^{D}\right) .
$$

The fourth-order contribution to the dipolar energy is

$$
U_{4, D}=\frac{75 \mathcal{K}_{D}}{16} \sum_{i, j} \frac{\left(z_{i}-z_{j}\right)^{4}}{\left|\mathbf{r}_{i}-\mathbf{r}_{j}\right|^{7}},
$$

which gets the form

$$
U_{4, D}=\Omega \frac{225 \mathcal{K}_{D}}{4 \sqrt{3}}|\psi|^{4} I_{4}^{D},
$$

with $I_{4}^{D}=3.410$. This leads to

$$
u=\frac{225}{4 \sqrt{3}} I_{4}^{D} .
$$

The sixth-order contribution to the dipolar energy is

$$
U_{6, D}=-\frac{245 \mathcal{K}_{D}}{32} \sum_{i, j} \frac{\left(z_{i}-z_{j}\right)^{6}}{\left|\mathbf{r}_{i}-\mathbf{r}_{j}\right|^{9}},
$$

which gives

$$
U_{6, D}=\Omega \frac{245 \mathcal{K}_{D}}{8 \sqrt{3}}|\psi|^{6}[\cos (6 \theta)-10] I_{6}^{D} .
$$

This is the lowest-order term that depends on $\theta$, as expected from symmetry, and defines the coefficients

$$
w=\frac{245}{8 \sqrt{3}} I_{6}^{D}
$$

and

$$
v=-\frac{2450}{8 \sqrt{3}} I_{6}^{D},
$$

where numerical evaluation yields $I_{6}^{D}=-2.537$. The gradient term is of a similar form as in Eq. (A34), and its explicit form reads

$$
U_{\nabla, D}=\gamma_{D} \Omega \frac{9 \mathcal{K}_{D}}{2}|\nabla \psi|^{2}
$$


where

$$
\begin{aligned}
\gamma_{D} & =-\sum_{n_{1}, n_{2}} \frac{\cos \left[\mathbf{K} \cdot \mathbf{r}_{n_{1}, n_{2}}\right]}{4 \sqrt{3}\left|\mathbf{r}_{n_{1}, n_{2}}\right|^{3}} \\
& =0.3366 .
\end{aligned}
$$

\section{APPENDIX B: EFFECTIVE THEORY FOR PHASE FLUCTUATIONS}

In the $Z_{6}$ ordered phase, the effective theory describing the phase fluctuations $\theta(\mathbf{r})$ can be approximated by a massive quadratic form characterized by a mass $m$. In this appendix, we derive this effective theory, allowing us to express the physical observables $h$ [Eq. (4.2)] and $C(\mathbf{r})$ [Eq. (4.14)] in terms of $\theta$-correlation functions. To find concrete expressions for these quantities as functions of $T$, we derive a self-consistent expression for $m$ using a variational principle. In particular, this provides its $T$ dependence, generated by fluctuation corrections.

Our starting point is the Euclidean action, which reads

$$
\begin{aligned}
& S=\int_{0}^{1 / T} d \tau \int_{\Omega} d^{2} r \mathcal{L}, \\
& \mathcal{L}=\frac{\rho_{s}}{2}\left\{|\nabla \theta|^{2}+\frac{1}{c^{2}}\left|\partial_{\tau} \theta\right|^{2}\right\}+h_{6} \cos 6 \theta,
\end{aligned}
$$

where $\tau$ denotes imaginary time and $\Omega$ the spatial area. Here, $c$ is the speed of sound, given by Eq. (2.31), and $\rho_{s}$ is the renormalized stiffness, which in the ordered phase is approximately equal to the bare stiffness, Eq. (3.4). Defining the Fourier components

$$
\theta_{q}=\int_{0}^{1 / T} d \tau \int d^{2} r e^{-i\left(\mathbf{k} \cdot \mathbf{r}+\omega_{n} \tau\right)} \theta(\mathbf{r}, \tau)
$$

where $q \equiv\left(\omega_{n}, c \mathbf{k}\right)$ with $\omega_{n}=2 \pi n T$ the Matsubara frequencies, the first (free) term in Eq. (B1) is recast as

$$
S_{\text {free }}=\frac{T \rho_{s}}{2 c^{2}} \sum_{\omega_{n}} \int \frac{d^{2} k}{(2 \pi)^{2}} q^{2}\left|\theta_{q}\right|^{2},
$$

while the second (clock) term cannot be written in a simple form. However, within the ordered phase (dark shaded region in Fig. 2), this term is relevant and generates a gap, which can be approximated by a mass-term correction to Eq. (B3).

We therefore introduce a variational ansatz for this massive theory in the form

$$
S_{0}=\frac{T}{2 \Omega} \sum_{q, q^{\prime}} G_{q, q^{\prime}}^{-1} \delta \theta_{q} \delta \theta_{q^{\prime}},
$$

where the propagator $G_{q, q^{\prime}}$ assumes the form

$$
G_{q, q^{\prime}}=\frac{T}{\Omega}\left\langle\delta \theta_{q} \delta \theta_{q^{\prime}}\right\rangle=\delta_{q,-q^{\prime}} \frac{c^{2}}{\rho_{s}\left(q^{2}+m^{2}\right)},
$$

yielding

$$
S_{0}=\frac{T \rho_{s}}{2 c^{2}} \sum_{\omega_{n}} \int \frac{d^{2} k}{(2 \pi)^{2}}\left(q^{2}+m^{2}\right)\left|\delta \theta_{q}\right|^{2} .
$$

Here, $\delta \theta$ denotes a deviation from the configuration $\left(\theta_{0}\right)$ minimizing the clock term, and $m$ is a variational parameter, subsequently adjusted to minimize the free energy. Employing the exact expression

$$
F=F_{0}-T \ln \left[\left\langle e^{-\left(S-S_{0}\right)}\right\rangle_{0}\right]
$$

where $F_{0}$ and the expectation value $\langle\ldots\rangle_{0}$ are evaluated with respect to $S_{0}$ [Eq. (B6)], and the convexity relation [71]

$$
\left\langle e^{-\left(S-S_{0}\right)}\right\rangle \geq e^{-\left\langle S-S_{0}\right\rangle},
$$

we find that $F \leq F_{\text {var }}$, where

$$
F_{\mathrm{var}}=F_{0}+T\left\langle S-S_{0}\right\rangle_{0}
$$

The minimum of $F_{\text {var }}$ with respect to the variational ansatz $S_{0}$ is therefore an optimal approximation of the free energy $F$. The condition for a minimum yields

$$
\frac{\partial F_{\mathrm{var}}}{\partial G(q)}=\frac{\partial F_{0}}{\partial G(q)}+T \frac{\partial\langle S\rangle_{0}}{\partial G(q)}=0
$$

[where $G(q)=G_{q, q}$ ] for all $q$. Employing Eq. (B6), we obtain

$$
\begin{aligned}
F_{0} & =-T \sum_{q}^{\prime} \ln [G(q)], \\
\langle S\rangle_{0} & =\frac{T}{\Omega}\left[\frac{1}{2 c^{2}} \sum_{q} \rho_{s} q^{2} G(q)+h_{6}\langle\cos (6 \theta)\rangle_{0}\right],
\end{aligned}
$$

where $\sum_{q}^{\prime} \equiv \sum_{k_{x}, k_{y}} \sum_{\omega_{n}>0}$, thus avoiding double counting. When substituted into Eq. (B10), one gets a self-consistent equation for the variational Green's function $G(q)$ :

$$
G^{-1}(q)=\frac{\rho_{s} q^{2}}{c^{2}}+2 h_{6} \frac{\partial\langle\cos (6 \theta)\rangle_{0}}{\partial G(q)} .
$$

Recalling that $\theta=\theta_{0}+\delta \theta$, where $\theta_{0}$ is one of the minima of the clock term [i.e., $\left.\left\langle\cos \left(6 \theta_{0}\right)\right\rangle_{0}=-\operatorname{sign}\left(h_{6}\right)\right]$, and using the quadratic form of $S_{0}$, the expectation value $\langle\cos (\beta \theta)\rangle_{0}$ for arbitrary $\beta$ can be expressed as 


$$
\begin{aligned}
\langle\cos (\beta \delta \theta)\rangle & =e^{-\left(\beta^{2} / 2\right)\left\langle(\delta \theta)^{2}\right\rangle}, \\
\left\langle(\delta \theta)^{2}\right\rangle & =\frac{T}{\Omega} \sum_{q} G(q) .
\end{aligned}
$$

For $\beta=6$, this yields

$$
\langle\cos (6 \theta)\rangle_{0}=-\operatorname{sign}\left(h_{6}\right) e^{-18\left\langle(\delta \theta)^{2}\right\rangle}
$$

Finally, employing $G^{-1}(q)=\rho_{s}\left(q^{2}+m^{2}\right) / c^{2}$ [Eq. (B5)] and inserting Eq. (B14) into Eq. (B12), we arrive at a selfconsistent equation for the mass $m$ :

$$
\begin{aligned}
m^{2} & \left.=\frac{36 c^{2}\left|h_{6}\right|}{\rho_{s}} \exp \left\{-18\left\langle(\delta \theta)^{2}\right\rangle\right\}\right\}, \\
\left\langle(\delta \theta)^{2}\right\rangle & =\frac{T}{\Omega} \sum_{q} \frac{c^{2}}{\rho_{s}\left(q^{2}+m^{2}\right)} .
\end{aligned}
$$

The last sum can be evaluated for arbitrary $T$ :

$$
\begin{aligned}
\left\langle(\delta \theta)^{2}\right\rangle & =\frac{c^{2}}{4 \pi \rho_{s}} \int_{0}^{\Lambda} \frac{d k k}{\sqrt{c^{2} k^{2}+m^{2}}} \operatorname{coth}\left(\frac{\sqrt{c^{2} k^{2}+m^{2}}}{2 T}\right) \\
& =\eta(T)\left\{\ln \sinh \left(\frac{c \Lambda}{2 T}\right)-\ln \sinh \left(\frac{m}{2 T}\right)\right\},
\end{aligned}
$$

where $\eta(T)$ is given by Eq. (3.8), and $\Lambda$ is the ultraviolet (UV) cutoff. Inserting this expression (using $c \Lambda \gg T$ ) into the first line of Eq. (B13) with $\beta=1$, and employing Eq. (4.2), we thus obtain the expression Eq. (4.3) for the layer separation $h$ as a function of $T$ for arbitrary $T<T_{6}$ and $r<r_{c}$. Similarly, substitution of Eq. (B16) into Eq. (B15) results in

$$
m^{2}=m_{0}^{2} \exp \{18 \eta(T) \ln [2 \sinh (m / 2 T)]\},
$$

where

$$
m_{0}^{2}=\left(36\left|h_{6}\right| c^{2} / \rho_{s}\right) e^{-9 c \Lambda / 2 \pi \rho_{s}}
$$

is $T$ independent. The self-consistent solution of Eq. (B17) yields the $T$-dependent mass $m(T)$. In the low- $T$ regime $T \ll m$ (below the dashed line in Fig. 2), one obtains a constant and $m \approx m_{0}$. However, in the classically ordered regime $m \ll T \ll T_{6}$, we get

$$
m(T) \approx T\left(\frac{m_{0}}{T}\right)^{\left[T_{6} /\left(T_{6}-T\right)\right]},
$$

where we have used $T_{6}=\left(2 \pi \rho_{s} / 9\right)$ (see Sec. III A).

\section{APPENDIX C: EVALUATION OF THE STRUCTURE FORM FACTOR}

To obtain the diffraction intensity $I_{\mathbf{k}_{3 d}}$ from Eq. (4.12), we recall the definition of the structure factor

$$
S_{\mathbf{k}_{3 d}}=\sum_{i} e^{i\left(\mathbf{k} \cdot \mathbf{r}_{\mathbf{i}}+k_{z} z_{i}\right)}
$$

Inserting this into Eq. (4.12), one obtains

$$
I_{\mathbf{k}_{3 d}}=\sum_{i, j} e^{i \mathbf{k} \cdot\left(\mathbf{r}_{\mathbf{i}}-\mathbf{r}_{\mathbf{j}}\right)}\left\langle e^{i k_{z}\left(z_{i}-z_{j}\right)}\right\rangle .
$$

For $k_{z} h \ll 1$, the last exponent can be expanded, yielding

$$
I_{\mathbf{k}_{3 d}}=I_{\left(\mathbf{k}, k_{z}=0\right)}+F\left(\mathbf{k}, k_{z}\right),
$$

with the leading $k_{z}$-dependent contribution

$$
F\left(\mathbf{k}, k_{z}\right)=k_{z}^{2} \sum_{i, j} e^{i \mathbf{k} \cdot\left(\mathbf{r}_{\mathbf{i}}-\mathbf{r}_{\mathbf{j}}\right)}\left\langle z_{i} z_{j}\right\rangle,
$$

where we have omitted a term $\propto \delta(\mathbf{k})$. Employing Eq. (2.8), we express the heights $z_{i}$ as

$z_{i}=\frac{\left|\psi_{0}\right|}{2}\left(e^{i\left(\theta_{0}+\mathbf{K} \cdot \mathbf{r}_{\mathbf{i}}\right)} e^{i \delta \theta\left(\mathbf{r}_{\mathbf{i}}\right)}+e^{-i\left(\theta_{0}+\mathbf{K} \cdot \mathbf{r}_{\mathbf{i}}\right)} e^{-i \delta \theta\left(\mathbf{r}_{\mathbf{i}}\right)}\right)$,

where $\mathbf{K}$ is given by Eq. (2.6). When substituted in Eq. (C4), one obtains a summation over terms with phase factors $e^{ \pm i \mathbf{K} \cdot\left(\mathbf{r}_{\mathbf{i}}+\mathbf{r}_{\mathbf{j}}\right)}$ or $e^{ \pm i \mathbf{K} \cdot\left(\mathbf{r}_{\mathbf{i}}-\mathbf{r}_{\mathbf{j}}\right)}$. Performing the sum over the center-of-mass coordinates first, the former type yields zero; from the latter type, we get $F\left(\mathbf{k}, k_{z}\right)$ of the form Eq. (4.14), which relates it to the correlation function $C(\mathbf{r})$.

We now evaluate $C(\mathbf{r})$ within the Gaussian theory for the fluctuating field $\delta \theta$ derived in Appendix B, which relates it to the correlation function $\langle\delta \theta(\mathbf{r}) \delta \theta(0)\rangle$ :

$$
C(\mathbf{r})=\langle\cos (\delta \theta)\rangle^{2} e^{\langle\delta \theta(\mathbf{r}) \delta \theta(0)\rangle},
$$

where we have used Eq. (B13) for $\langle\cos (\delta \theta)\rangle$. Inside the ordered phase, the Gaussian theory for $\delta \theta$ is massive, and the propagator in momentum space is given by Eq. (B5), with $m$ the mass associated with the clock-ordering term, evaluated as a function of $T$ and the parameters of the model using a variational principle in Appendix B. We thus obtain

$$
\begin{aligned}
\langle\delta \theta(\mathbf{r}) \delta \theta(0)\rangle & =\frac{T}{\Omega} \sum_{q} e^{i \mathbf{k} \cdot \mathbf{r}} G_{q, q} \\
& =\frac{c^{2}}{4 \pi \rho_{s}} \int_{0}^{\Lambda} \frac{d k k J_{0}(k|\mathbf{r}|)}{\sqrt{c^{2} k^{2}+m^{2}}} \operatorname{coth}\left(\frac{\sqrt{c^{2} k^{2}+m^{2}}}{2 T}\right) .
\end{aligned}
$$


From this general expression, one can obtain asymptotic expressions for $\langle\delta \theta(\mathbf{r}) \delta \theta(0)\rangle$ and, consequently, $C(\mathbf{r})$ for large $|\mathbf{r}|$ in two limits-for $T$ above or below the crossover line:

$$
\begin{array}{ll}
\langle\delta \theta(\mathbf{r}) \delta \theta(0)\rangle \approx \frac{c}{4 \pi \rho_{s}|\mathbf{r}|} e^{-m|\mathbf{r}| / c} & (T \ll m), \\
\langle\delta \theta(\mathbf{r}) \delta \theta(0)\rangle \approx \eta(T) K_{0}\left(\frac{m|\mathbf{r}|}{c}\right) & \left(m \ll T<T_{6}\right),
\end{array}
$$

with $K_{0}(z)$ the modified Bessel function. Inserting this into Eq. (C6), this yields corresponding expressions for $C(\mathbf{r})$. The scale of exponential decay in both expressions, $c / m$, dictates the width of the Bragg peaks.

[1] J. M. Kosterlitz and D. J. Thouless, Ordering, Metastability and Phase Transitions in Two-Dimensional Systems, J. Phys. C 6, 1181 (1973).

[2] V.L. Berezinskii, Destruction of Long-range Order in One-Dimensional and Two-Dimensional Systems Possessing a Continuous Symmetry Group. I. Classical Systems, Sov. Phys. JETP 32, 493 (1971); II. Quantum Systems, Sov. Phys. JETP 34, 610 (1972).

[3] J. V. José, L. P. Kadanoff, S. Kirkpatrick, and D. R. Nelson, Renormalization, Vortices, and Symmetry-Breaking Perturbations in the Two-Dimensional Planar Model, Phys. Rev. B 16, 1217 (1977).

[4] M. Oshikawa, Ordered Phase and Scaling in $Z_{n}$ Models and the Three-State Antiferromagnetic Potts Model in Three Dimensions, Phys. Rev. B 61, 3430 (2000).

[5] J. Lou, A. W. Sandvik, and L. Balents, Emergence of U(1) Symmetry in the $3 D X Y$ Model with $Z_{q}$ Anisotropy, Phys. Rev. Lett. 99, 207203 (2007).

[6] J. Hove and A. Sudbø, Criticality Versus $q$ in the $(2+1)$ Dimensional $Z_{q}$ Clock Model, Phys. Rev. E 68, 046107 (2003).

[7] D. Blankschtein, M. Ma, A. N. Berker, G. S. Grest, and C. M. Soukoulis, Orderings of a Stacked Frustrated Triangular System in Three Dimensions, Phys. Rev. B 29, 5250 (1984).

[8] P. M. Chaikin and T. C. Lubensky, Principles of Condensed Matter Physics (Cambridge University Press, Cambridge, England, 2000).

[9] D. J. Bishop and J. D. Reppy, Study of the Superfluid Transition in Two-Dimensional ${ }^{4} \mathrm{He}$ Films, Phys. Rev. Lett. 40, 1727 (1978).

[10] A. F. Hebard and A. T. Fiory, Evidence for the KosterlitzThouless Transition in Thin Superconducting Aluminum Films, Phys. Rev. Lett. 44, 291 (1980).

[11] B. I. Halperin and D. R. Nelson, Theory of TwoDimensional Melting, Phys. Rev. Lett. 41, 121 (1978); D. R. Nelson and B. I. Halperin, Dislocation-Mediated Melting in Two Dimensions, Phys. Rev. B 19, 2457 (1979).

[12] S. T. Chui, Grain-Boundary Theory of Melting in Two Dimensions, Phys. Rev. B 28, 178 (1983).
[13] B. K. Clark, M. Casula, and D. M. Ceperley, Hexatic and Mesoscopic Phases in a 2D Quantum Coulomb System, Phys. Rev. Lett. 103, 055701 (2009).

[14] G. M. Bruun and D. R. Nelson, Quantum Hexatic Order in Two-Dimensional Dipolar and Charged Fluids, Phys. Rev. B 89, 094112 (2014).

[15] R. A. Quinn and J. Goree, Experimental Test of TwoDimensional Melting through Disclination Unbinding, Phys. Rev. E 64, 051404 (2001); O. F. Petrov, M. M. Vasiliev, Ye Tun, K. B. Statsenko, O.S. Vaulina, E. V. Vasilieva, and V.E. Fortov, Two-Dimensional Phase Transition in a Strongly Nonideal Dusty Plasma, JETP 120, 327 (2015).

[16] Z. Hadzibabic, P. Krüger, M. Cheneau, B. Battelier, and J. Dalibard, Berezinskii-Kosterlitz-Thouless Crossover in a Trapped Atomic Gas, Nature (London) 441, 1118 (2006); P. Krüger, Z. Hadzibabic, and J. Dalibard, Critical Point of an Interacting Two-Dimensional Atomic Bose Gas, Phys. Rev. Lett. 99, 040402 (2007).

[17] A. Polkovnikov, E. Altman, and E. Demler, Interference between Independent Fluctuating Condensates, Proc. Natl. Acad. Sci. U.S.A. 103, 6125 (2006).

[18] V. Schweikhard, S. Tung, and E. A. Cornell, Vortex Proliferation in the Berezinskii-Kosterlitz-Thouless Regime on a Two-Dimensional Lattice of Bose-Einstein Condensates, Phys. Rev. Lett. 99, 030401 (2007).

[19] R. G. Melko, A. Paramekanti, A. A. Burkov, A. Vishwanath, D. N. Sheng, and L. Balents, Supersolid Order from Disorder: Hard-Core Bosons on the Triangular Lattice, Phys. Rev. Lett. 95, 127207 (2005).

[20] D. Heidarian and K. Damle, Persistent Supersolid Phase of Hard-Core Bosons on the Triangular Lattice, Phys. Rev. Lett. 95, 127206 (2005).

[21] S. Wessel and M. Troyer, Supersolid Hard-Core Bosons on the Triangular Lattice, Phys. Rev. Lett. 95, 127205 (2005).

[22] K. Damle, Melting of Three-Sublattice Order in Easy-Axis Antiferromagnets on Triangular and Kagome Lattices, Phys. Rev. Lett. 115, 127204 (2015); D. Heidarian and K. Damle, Two-Step Melting of Three-Sublattice Order in $\mathrm{S}=1$ Easy-Axis Triangular Lattice Antiferromagnets, arXiv:1512.01346.

[23] S. Elitzur, R. B. Pearson, and J. Shigemitsu, Phase Structure of Discrete Abelian Spin and Gauge Systems, Phys. Rev. D 19, 3698 (1979).

[24] A. Ukawa, P. Windey, and A. H. Guth, Dual Variables for Lattice Gauge Theories and the Phase Structure of $Z(N)$ Systems, Phys. Rev. D 21, 1013 (1980).

[25] D. H.E. Dubin and T.M. ONeil, Trapped Nonneutral Plasmas, Liquids, and Crystals (the Thermal Equilibrium States), Rev. Mod. Phys. 71, 87 (1999).

[26] J. P. Schiffer, Order in Confined Ions, J. Phys. B 36, 511 (2003).

[27] E. Wigner, On the Interaction of Electrons in Metals, Phys. Rev. 46, 1002 (1934).

[28] E. Rousseau, D. Ponarin, L. Hristakos, O. Avenel, E. Varoquaux, and Yu. Mukharsky, Addition Spectra of Wigner Islands of Electrons on Superfluid Helium, Phys. Rev. B 79, 045406 (2009).

[29] H. M. Van Horn, Dense Astrophysical Plasmas, Science 252, 384 (1991). 
[30] D. E. Winget and S. O. Kepler, Pulsating White Dwarf Stars and Precision Asteroseismology, Annu. Rev. Astron. Astrophys. 46, 157 (2008).

[31] Ion Traps for Tomorrow's Applications, edited by M. Knoop, I. Marzoli, and G. Morigi, Proceedings of the International School of Physics "Enrico Fermi" (IOS Press, Amsterdam, 2015), Vol. 189.

[32] D. H. E. Dubin, Correlation Energies of Simple Bounded Coulomb Lattices, Phys. Rev. A 40, 1140 (1989); D. H. E. Dubin, Theory of Structural Phase Transitions in a Trapped Coulomb Crystal, Phys. Rev. Lett. 71, 2753 (1993).

[33] R. W. Hasse and J.P. Schiffer, The Structure of the Cylindrically Confined Coulomb Lattice, Ann. Phys. (N.Y.) 203, 419 (1990).

[34] G. Birkl, S. Kassner, and H. Walther, Multiple-Shell Structures of Laser-Cooled ${ }^{24} \mathrm{Mg}^{+}$Ions in a Quadrupole Storage Ring, Nature (London) 357, 310 (1992); I. Waki, S. Kassner, G. Birkl, and H. Walther, Observation of Ordered Structures of Laser-Cooled Ions in a Quadrupole Storage Ring, Phys. Rev. Lett. 68, 2007 (1992).

[35] W. M. Itano, J. J. Bollinger, J. N. Tan, B. Jelenković, X. P. Huang, and D. J. Wineland, Bragg Diffraction from Crystallized Ion Plasmas, Science 279, 686 (1998).

[36] T. B. Mitchell, J. J. Bollinger, D. H. E. Dubin, X.-P. Huang, W. M. Itano, and R. H. Baughman, Direct Observations of Structural Phase Transitions in Planar Crystallized Ion Plasmas, Science 282, 1290 (1998); T. B. Mitchell, J. J. Bollinger, X.-P. Huang, W. M. Itano, and D. H. E. Dubin, Direct Observations of the Structural Phases of Crystallized Ion Plasmas, Phys. Plasmas 6, 1751 (1999).

[37] B. C. Sawyer, J. W. Britton, A. C. Keith, C.-C. J. Wang, J. K. Freericks, H. Uys, M. J. Biercuk, and J. J. Bollinger, Spectroscopy and Thermometry of Drumhead Modes in a Mesoscopic Trapped-Ion Crystal Using Entanglement, Phys. Rev. Lett. 108, 213003 (2012); B. C. Sawyer, J. W. Britton, and J. J. Bollinger, Spin Dephasing as a Probe of Mode Temperature, Motional State Distributions, and Heating Rates in a Two-Dimensional Ion Crystal, Phys. Rev. A 89, 033408 (2014).

[38] N. Kjærgaard and M. Drewsen, Observation of a Structural Transition for Coulomb Crystals in a Linear Paul Trap, Phys. Rev. Lett. 91, 095002 (2003).

[39] S. Fishman, G. De Chiara, T. Calarco, and G. Morigi, Structural Phase Transitions in Low-Dimensional Ion Crystals, Phys. Rev. B 77, 064111 (2008).

[40] E. Shimshoni, G. Morigi, and S. Fishman, Quantum Zigzag Transition in Ion Chains, Phys. Rev. Lett. 106, 010401 (2011).

[41] E. Shimshoni, G. Morigi, and S. Fishman, Quantum Structural Phase Transition in Chains of Interacting Atoms, Phys. Rev. A 83, 032308 (2011).

[42] S. Fishman, D. Podolsky, E. Shimshoni, P. Silvi, T. Calarco, S. Montangero, and G. Morigi, The Linear-Zigzag Phase Transition for Cold Ion Chains, Proceedings of the International School of Physics "Enrico Fermi" (IOS Press, Amsterdam, 2015), Vol. 189, pp. 103-114.

[43] P. Silvi, G. De Chiara, T. Calarco, G. Morigi, and S. Montangero, Full Characterization of the Quantum Linear-Zigzag Transition in Atomic Chains, Ann. Phys. (Amsterdam) 525, 827 (2013).
[44] P. Silvi, T. Calarco, G. Morigi, and S. Montangero, Ab Initio Characterization of the Quantum Linear-Zigzag Transition Using Density Matrix Renormalization Group Calculations, Phys. Rev. B 89, 094103 (2014).

[45] J. M. Taylor and T. Calarco, Wigner Crystals of Ions as Quantum Hard Drives, Phys. Rev. A 78, 062331 (2008).

[46] M. J. Biercuk, H. Uys, A. P. VanDevender, N. Shiga, W. M. Itano, and J. J. Bollinger, Optimized Dynamical Decoupling in a Model Quantum Memory, Nature (London) 458, 996 (2009).

[47] Eugene Demler (private communication).

[48] E. Fradkin and L. P. Kadanoff, Disorder Variables and Para-fermions in Two-Dimensional Statistical Mechanics, Nucl. Phys. B170, 1 (1980).

[49] S. Sachdev, Quantum Phase Transitions (Cambridge University Press, Cambridge, England, 1999).

[50] A dangerously irrelevant variable is one that is irrelevant at the critical point but which nevertheless cannot be set to zero in the effective Hamiltonian. A possible scenario is that slightly away from the critical point, the flow of its coupling constant under the renormalization group is initially decreasing but starts to grow at some longer length scale; see, e.g., Fig. 1 in Ref. [4]. In our case, the clock term $h_{6}$ becomes relevant immediately upon entering the ordered phase at $T=0$ and is responsible for pinning $\theta$ into one of six allowed values.

[51] T. Lahaye, C. Menotti, L. Santos, M. Lewenstein, and T. Pfau, The Physics of Dipolar Bosonic Quantum Gases, Rep. Prog. Phys. 72, 126401 (2009).

[52] M. Knap, E. Berg, M. Ganahl, and E. Demler, Clustered Wigner-Crystal Phases of Cold Polar Molecules in Arrays of One-Dimensional Tubes, Phys. Rev. B 86, 064501 (2012).

[53] D. Podolsky, E. Shimshoni, P. Silvi, S. Montangero, T. Calarco, G. Morigi, and S. Fishman, From Classical to Quantum Criticality, Phys. Rev. B 89, 214408 (2014).

[54] H. Kadau, M. Schmitt, M. Wenzel, C. Wink, T. Maier, I. Ferrier-Barbut, and T. Pfau, Observing the Rosensweig Instability of a Quantum Ferrofluid, Nature (London) 530, 194 (2016).

[55] A. I. Larkin and S. A. Pikin, Phase Transitions of the First Order but Nearly of the Second, Zh. Eksp. Teor. Fiz. 56, 1664 (1969) [Sov. Phys. JETP 29, 891 (1969)].

[56] F. Cartarius, G. Morigi, and A. Minguzzi, Structural Transitions of Nearly Second Order in Classical Dipolar Gases, Phys. Rev. A 90, 053601 (2014).

[57] M. E. Fisher, M. N. Barber, and D. Jasnow, Helicity Modulus, Superfluidity, and Scaling in Isotropic Systems, Phys. Rev. A 8, 1111 (1973).

[58] S. Sachdev, Universal Relaxational Dynamics Near Two-Dimensional Quantum Critical Points, Phys. Rev. B 59, 14054 (1999).

[59] M.E. Fisher and P. Pfeuty, Critical Behavior of the Anisotropic n-vector Model, Phys. Rev. B 6, 1889 (1972).

[60] M. Campostrini, M. Hasenbusch, A. Pelissetto, P. Rossi, and E. Vicari, Critical Behavior of the Three-Dimensional XY Universality Class, Phys. Rev. B 63, 214503 (2001).

[61] J. M. Caillol, D. Levesque, J. J. Weis, and J. P. Hansen, A Monte-Carlo Study of the Classical Two-Dimensional OneComponent Plasma, J. Stat. Phys. 28, 325 (1982). 
[62] J. Eschner, G. Morigi, F. Schmidt-Kaler, and R. Blatt, Laser Cooling of Trapped Ions, J. Opt. Soc. Am. B 20, 1003 (2003).

[63] R. Lechner, C. Maier, C. Hempel, P. Jurcevic, B. P. Lanyon, T. Monz, M. Brownnutt, R. Blatt, and C. F. Roos, Electromagnetically-Induced-Transparency Ground-State Cooling of Long Ion Strings, Phys. Rev. A 93, 053401 (2016),

[64] M. Brownnutt, M. Kumph, P. Rabl, and R. Blatt, Ion-Trap Measurements of Electric-Field Noise Near Surfaces, Rev. Mod. Phys. 87, 1419 (2015).

[65] H. Weber and P. Minnhagen, Monte Carlo Determination of the Critical Temperature for the Two-Dimensional XY Model, Phys. Rev. B 37, 5986(R) (1988).

[66] A. Dantan, J. P. Marler, M. Albert, D. Guénot, and M. Drewsen, Noninvasive Vibrational Mode Spectroscopy of Ion Coulomb Crystals through Resonant Collective
Coupling to an Optical Cavity Field, Phys. Rev. Lett. 105, 103001 (2010).

[67] G. De Chiara, T. Calarco, S. Fishman, and G. Morigi, Ramsey Interferometry with a Spin Embedded in a Coulomb Chain, Phys. Rev. A 78, 043414 (2008).

[68] A. Retzker, R. C. Thompson, D. M. Segal, and M. B. Plenio, Double Well Potentials and Quantum Phase Transitions in Ion Traps, Phys. Rev. Lett. 101, 260504 (2008).

[69] M. Knap, A. Kantian, T. Giamarchi, I. Bloch, M. D. Lukin, and E. Demler, Probing Real-Space and Time-Resolved Correlation Functions with Many-Body Ramsey Interferometry, Phys. Rev. Lett. 111, 147205 (2013).

[70] I. Bloch, J. Dalibard, and W. Zwerger, Many-Body Physics with Ultracold Gases, Rev. Mod. Phys. 80, 885 (2008).

[71] See, e.g., R. P. Feynman, Statistical Mechanics: A Set of Lectures (Addison-Wesley, Boston, 1972). 